H. Umemura

Nagoya Math. J.

Vol. 133 (1994), 1-55

\title{
ON A CLASS OF NUMBERS GENERATED BY DIFFERENCIAL EQUATIONS RELATED WITH ALGEBRAIC GROUPS
}

\author{
HIROSHI UMEMURA
}

\section{Introduction}

In this paper we propose a new category $\mathbf{Q}^{\text {cl }}$ of complex numbers which contains $\pi, e$ and the set of algebraic numbers. In fact this category contains most of the numbers studied so far in number theory. An element of the category is here called a classical number. The category of the classical numbers forms an algebraically closed field and consists of countably many numbers. The definition depends on algebraic differential equations related with algebraic groups. Throughout the paper unless otherwise stated, we deal with functions of one variable and a differential equation is an ordinary differential equation. We are inspired of the Leçons de Stockholm of Painlevé [P]. His objective was to discover new transcendental functions defined by algebraic differential equations generalizing the Weierstrass $\wp$-function. To this end there are two major tasks to be done. The first is to find candidates of algebraic differential equations which may define new functions. We are concerned with the second which is to check whether the candidates really define new functions. So he introduced a class of functions inductively defined from the field $\mathbf{C}(x)$ of the rational functions by admissible operations. In [U4], [U6] we analyzed his operations and introduced the permissible operations $(\mathrm{O}),(\mathrm{P} 1),(\mathrm{P} 2), \ldots$, (P5) (cf. $\S 1$ ). We proved that allowing the permissible operations is equivalent to admitting $G$-primitive extensions of differential fields in the language of Kolchin. We defined the field of the classical functions as a field of the meromorphic functions obtained from the field $\mathbf{C}(x)$ of the rational functions by a finite iteration of permissible operations (cf. [U4]). All the functions related with differential equations in [WW] are classical in this sense, for example exp $x$, $\log x$, the hypergeometric functions, the elliptic functions and so on. The irreducibility theorem says that we can not solve the first differential equation of Painlevé starting from the field $\mathbf{C}(X)$ of the rational functions by any finite iteration of the

Received July 8, 1992. 
permissible operations or equivalently no classical function is a solution of the first differential equation of Painlevé ([U2]).

It is more natural however to start from $\mathbf{Q}(x)$ rather than from $\mathbf{C}(x)$ and allow in each step of the inductive construction, the classical functions dependent on an algebraic group over a field of so far known numbers (= inductively so far introduced constant functions) whose Laurent coefficients with respect to $x-a$ are known numbers. Here the number $a$ is a suitable so far known number (Definition (2.4)). For example, at the first step of construction, we allow intuitively an extension field $\mathbf{Q}(x, v) / \mathbf{Q}(x)$ of functions satisfying the following conditions: (i) There exists an algebraic group $G$ defined over $\mathbf{Q}$ such that $v$ is $G$-primitive over $\mathbf{Q}(x)$; (ii) There exists a rational number $a$ such that every function in $\mathbf{Q}(x, v)$ has a rational Laurent coefficients with respect to a local parameter $x-a$. In particular if we take the multiplicative group $\mathbf{G}_{m \mathbf{Q}}$ as the algebraic group $\mathrm{G}$, it yields a quite concrete example which illustrates our defininion. In this case the following extension field $\mathbf{Q}(x, v) / \mathbf{Q}(x)$ of functions is allowed as a first step: (i) The function $v=v(x)$ satisfies a differential equation $v^{\prime}(x)=$ $a(x) v(x)$ for a suitable rational function $a(x) \in \mathbf{Q}(x)$; (ii) There exists a rational number $a$ such that the rational function $a(x)$ is regular at the point $x=a$ and such that the value $v(a)$ of the function $v(x)$ at $a$ is rational. Among the Q-classical functions, those which are constant seem to be important and are called classical numbers. The algebraic numbers are classical (Theorem (3.1)). $\pi$ and $\boldsymbol{e}$ are classical (Examples (2.12) and (2.14)). More generally for any rational number $r, \exp r$ and $\log r(r \neq 0)$ are classical (Examples (2.12) and (2.14)). Another interesting example is given by an elliptic curve $E$ defined over $\mathbf{Q}$. Let $\mathfrak{P}(x)$ be the Weierstrass $\varnothing$-function giving $E$. Then $\wp(r)$ is a classical number for any $r \in \mathbf{Q}$ if $\boldsymbol{r}$ is not a pole of $\mathfrak{P}(x)$ (Example (2.15)). Similarly we can construct classical numbers using the hypergeometric functions. The most general assertion is that the composite of two $\mathbf{Q}$-classical functions is $\mathbf{Q}$-classical and hence in particular for a $\mathbf{Q}$-classical function $f$ and z classical number $a$, the value $f(a)$ is a classical number if the function $f$ is regular at $a$ (Theorem (3.7) and Theorem (3.15)).

We show that if $a, b$ are classical numbers, the definite integral $\int_{a}^{b} f(x) d x$ of a Q-classical function $f(x)$ is a classical number (Theorem (3.8)). As a corollary of this we show in $\S 5$ that a period $\int_{r} \omega$ is classical for a 1 -form $\omega$ on an algebraic variety $V$ defined over a field $K$ of classical numbers and for $r \in$ $H_{1}\left(V \otimes_{K} \mathbf{C}, Z\right)$. 
It is J. Liouville (1844) who first recognized transcendental numbers. He was interested also in the generation of functions or the construction of functions from the known functions by admissible operations from the field $\mathbf{C}(x)$ of rational func tions. He considered generating functions as an analogue of the resolution of algebraic equations by radicals. Thus he introduced the rigorous defininion of elementary functions and proved among other things that the elliptic integral is not elementary ([L] and [R2]). In this definition too it is more natural to start from $\mathbf{Q}(x)$ than from $\mathbf{C}(x)$. Thus we arrive at the notion of $\mathbf{Q}$-elementary functions. A constant $\mathbf{Q}$-elementary function is called an elementary number. The field of elementary numbers is denoted by $\mathbf{Q}^{\text {el }}$. We prove that a $\mathbf{Q}$-elementary function is $\mathbf{Q}$-classical (Theorem (4.10)) and hence in particular $\mathbf{Q}^{\mathrm{el}} \subset \mathbf{Q}^{\mathrm{cl}}$. Every algebraic number, $\pi$ and $e$ are elementary. We have no example of a classical number which is not elementary (Problem (4.5)).

Extensive examples of $\mathbf{Q}$-classical functions are given in $§ 6$. Namely the hypergeometric functions, the Bessel functions, the Legendre functions, the Weierstrass functions and the Jacobian theta functions of 2 division points are Q-classical under certain conditions. The most interesting example is the gamma function $\Gamma(x)$ (Example (6.13)). Because as is well known, the gamma function does not satisfy any algebraic differential equation over $\boldsymbol{C}(x)$ and hence is not classical. But following an idea of Gauss $[G]$, we show that the values $\Gamma(r)$ is classical for $r \in \mathbf{Q}$ if $r \neq 0,-1,-2, \ldots$. Those examples illustrate our idea but they are not thoroughly analyzed and many natural questions arise from them such as whether the value $\Gamma(u)$ is classical for every classical number $u$ if $u \neq 0$, $-1,-2, \ldots$ (Problem (6.14)).

The most important problem is a construction of non-classical numbers, which seems difficult. If the field $\mathbf{Q}^{c 1}$ does not contain all the interesting numbers, it is because the algebraic differential equation is not the unique way of defining functions. The other possible definitions are for example, transcendental differential equations (une étude générale de telle équation serait prématurée, Leçons de Stockholm, 1985, Painlevé), difference equations, more generally functional equations and Taylor expansions or definition by series.

We would like to say a word about the relations of this paper with the works of precedent authors. We heard that once Mikio Sato had an idea of generating numbers by successive substitutions of known numbers in good functions. Noriko Hirata kindly indicated us the work of Mordukhai-Boltovski: Hypertranscendental functions and hypertranscendental numbers, Dokl. Akad. Nauk SSSR, 64 (1949), 21-24. In this article he introduced a notion of hypertranscendental numbers in order to classify transcendental numbers. A complex number is said to be hyper- 
transcendental if it can not be a value at an algebraic point of a function which is a solution of algebraic differential equation with coefficients in $\mathbf{Z}[x]$ and with algebraic initial conditions.

Our frame work is more natural and a systematic study is done. We restrict ourselves to algebraic differential equations related with algebraic groups. We generate functions and get as a natural object, the set of constant $\mathbf{Q}$-classical functions or the classical numbers rather than the values of thus obtained functions (Definition (2.10)). And later we study values of functions (Theorem (3.7)).

Our theory is developed in the frame work of algebraic geometry. It requires only basic knowledge of algebraic geometry which is found in $[\mathrm{S}]$ or $[\mathrm{H}]$. We hope however that our paper will interest number theorists and consider that there may be readers who are not familiar with algebraic geometry. So we added an Appendix in which we explained for non-specialists terminologies of algebraic geometry.

\section{§1. Primitive extensions}

We work in a differential field of the meromorphic functions over a domain $D$ of $\mathbf{C}$. Let $x$ be a coordinate system on $\mathbf{C}$ fixed once for all. In our papers [U2] and [U4], we introduced the permissible operations (O), (P1), (P2), ., (5). They are the operations allowed to use when we introduce a new meromorphic function on $D$ from a set of given meromorphic functions on $D$. Let us recall their definition.

(O) Differentiation $d / d x$. Given a meromorphic function on $D$, we can differentiate it.

(P1) Four rules of arithmetic; addition, subtraction, multiplication and division. Given two meromorphic functions $f, g$ on $D$, we can form $f+g, f-g, f g$ and $f / g$ if $g \neq 0$.

(P2) Solution of an algebraic equation. If $n$ meromorphic functions $a_{1}$, $a_{2}, \ldots, a_{n}$ on $D$ are given, we can introduce every solution $f$ of an algebraic equation

$$
f^{n}+a_{1} f^{n-1}+a_{2} f^{n-1}+\cdots+a_{n}=0 .
$$

(P3) Quadrature. Given a meromorphic function $f$ on $D$, we can find every indefinite integral $\int f d x$ or we can solve a differential equation $y^{\prime}=f(x)$. 
(P4) Solution of a linear ordinary differential equation. Given $n$ meromorphic functions $a_{1}, a_{2}, \ldots, a_{n}$ on $D$, we can introduce every solution $f$ of a linear differential equation

$$
\left((d / d x)^{n}+a_{1}(d / d x)^{n-1}+\cdots+a_{n}\right) f=0 .
$$

(P5) Substitution in abelian function. Let $\Gamma \subset \mathbf{C}^{n}$ be a lattice such that $\mathbf{C}^{n} / \Gamma$ is an abelian variety. We denote the projection $\mathbf{C}^{n} \rightarrow \mathbf{C}^{n} / \Gamma$ by $p$. Let $\varphi$ be a meromorphic function on $\mathbf{C}^{n} / \Gamma$ so that $\varphi \circ p: \mathbf{C}^{n} \cdots \rightarrow \mathbf{C}$ is an abelian function by definition. (We use a dotted arrow for a meromorphic map.) Suppose now that $n$ meromorphic functions $f_{1}, f_{2}, \ldots, f_{n}$ on $D$ are given. So we get a meromorphic map $F: D \cdots \rightarrow \mathbf{C}^{n}$ sending $x$ to $\left(f_{1}(x), f_{2}(x), \ldots, f_{n}(x)\right)$. We are allowed to introduce a composite function $D \stackrel{F}{*} \rightarrow \mathbf{C}^{n} \stackrel{p}{\rightarrow} \mathbf{C}^{n} / \Gamma \stackrel{\varphi}{\rightarrow} \rightarrow \mathbf{C}$ which is a meromorphic function on $D$. Namely we may substitute the given functions $f_{1}, f_{2}, \ldots, f_{n}$ in the abelian function $\varphi^{\circ} p$ to introduce the new meromorphic function $\varphi^{\circ} p^{\circ} F$ on $D$. Here we must assume that the image of $p \circ F$ is not contained in the polar divisor of the meromorphic function $\varphi$ so that the composite function $\varphi^{\circ} p^{\circ} F$ is defined.

Strictly speaking in the permissible operation (P2), we assume that the solution $f$ is meromorphic on $D$. Since we are interested in the field extension, we may replace $D$ by a smaller domain $D^{\prime}$ if necessary, so that all the solution of the algebraic equation are meromorphic on $D^{\prime}$. For this reason we need not be too nervous of the domain $D$. The same remark should be done for the operations (P3) and (P4).

We introduced a group theoretic operation (Q) in [U1]. Let us recall the definition of the operation (Q). For our purpose, we had better review the definition of $G$-primitive extension whose particular case is the operation (Q).

A differential field is a field $L$ together with derivation $\delta: L \rightarrow L$. So we have $\delta(a+b)=\delta(a)+\delta(b), \delta(a b)=\delta(a) b+a \delta(b)$ for $a, b \in L$. We assume that the field $L$ is of characteristic 0 . We assume that the field $L$ is of characteristic 0 . We sometimes denote $\delta a$ and $\delta \delta a=\delta^{2} a$ by $a^{\prime}$ and $a^{\prime \prime}$ respectively. More generally $\delta^{n} a$ will be denoted by $a^{(n)}$ for $n \in \mathbf{N}$. We say that an element $c$ of $L$ is constant if $\delta a=0$. The field of the constants of $L$ is denoted by $C_{L}$.

Let $(L, \delta)$ be a differential field and $C$ a subfield consisting of constants of $L$. Let $G$ be an algebraic group defined over $C$ and $\operatorname{Spec} L \stackrel{V}{\rightarrow} G_{L}=G \otimes_{C} L$ be an $L$-rational point. (We refer the reader to (A3), (A4) in Appendix if he is not familiar with algebraic geometry). We often confuse $v$ with its image. Let $U$ be an affine neighbourhood of the image point $\operatorname{Spec} L \stackrel{V}{\rightarrow} G_{L} \rightarrow G$ on $G$. Then $U \otimes_{C} L \subset G \otimes_{C} L$ 
is an affine neighbourhood of the $L$-rational point $v$.

Let $f: G_{L} \rightarrow G$ be the morphism of field extension (cf. (A3)). The image $f^{*} h$ is an element of $\Gamma\left(U_{L}, \mathscr{O}_{U_{L}}\right)$ for $h \in \Gamma\left(U, \mathscr{O}_{U}\right)$ and hence the value $\left(f^{*} h\right)(v)$ of the function at $v$ is in $L$. (Here $\mathscr{O}$ denotes the structure sheaf of $G$ and $\Gamma\left(U, \mathscr{O}_{U}\right)$ is a group of the sections of $\mathscr{O}_{U}$ over $U$ (cf. (A1) and (A2)). Hence we can apply $\delta$ on $\left(f^{*} h\right)(v) \in L$. Considering the sequence of mappings $\Gamma\left(U, \mathscr{O}_{U}\right) \stackrel{f^{*}}{\rightarrow} \Gamma\left(U_{L}, \mathscr{O}_{U_{L}}\right) \rightarrow$ $\hat{O}_{v} / m_{v}=L(v) \simeq L \stackrel{\delta}{\rightarrow} L$, let us put $\delta(v)(f)=\delta\left(\left(f^{*} h\right)(v)\right)$ (cf. (A2) and (A4)). Then the mapping $\Gamma\left(U, \mathcal{O}_{U}\right) \rightarrow L$ sending $f$ to $\delta(v)(f)$ is a $C$-derivation. This can be extended to a unique $L$-derivation $\Gamma\left(U, \mathscr{O}_{U}\right) \otimes_{C} L=\Gamma\left(U_{L}, \mathscr{O}_{U_{L}}\right) \rightarrow L$ at the point $v$ which we denote also be $\delta(v)$ : The map $\delta(v)$ is $L$-linear and $\delta(v)(f g)=$ $\delta(v)(f) g(v)+f(v) \delta(v)(g)$ for $f, g \in \Gamma\left(U_{L}, \mathscr{O}_{U_{L}}\right)$. Thus $\delta(v)$ is extended to a unique element of $\operatorname{Der}_{L}\left(\mathscr{O}_{G_{L} v}, L(v)\right)$, which is again denoted by $\delta(v),\left(\mathscr{O}_{G_{L} v}\right.$ is the fibre of the structure sheaf $\mathscr{O}_{G_{L}}$ at $v$ (cf. (A1)). Namely the derivation $\delta(v)$ is a tangent vector at $v$ on $G_{L}$. The above construction shows that the derivation $\delta(v): \mathscr{O}_{V} \rightarrow L(v)=L$ is independent of the choice of the affine open set $U$. There exists a unique right invariant derivation $l \delta(v) \in \operatorname{Lie} G_{L}=($ Lie $G) \otimes_{C} L$ on the algebraic group $G_{L}$ whose value at $v$ is $\delta(v)$ (Lie $G$ denotes the Lie algebra of $G$ or Lie $G$ is the $C$-vector space of the right invariant vector fields on $G$ ).

Let $D \subset \boldsymbol{C}$ be a complex domain, $L$ a field of meromorphic functions on $D$ containing the field $\mathbf{C}$ of constant functions and $V$ be an algebraic variety defined over C. Let $v: \operatorname{Spec} L \rightarrow V$ be an $L$-valued point. Let $U=\operatorname{Spec} \mathbf{C}\left[u_{1}, u_{2}, \ldots, u_{n}\right]$ be an affine neighbourhood of $v(\operatorname{Spec} L) \in V$. Then the point $v$ is defined by a C-morphism $\mathbf{C}\left[u_{1}, u_{2}, \ldots, u_{n}\right] \rightarrow L\left(u_{i} \rightarrow a_{i}\right.$ for $\left.1 \leq i \leq n\right)$ of commutative algebras. Hence it gives a meromorphic map $f(v): D \cdots \rightarrow \mathbf{C}^{n}, x \rightarrow\left(a_{1}(x), a_{2}(x), \ldots, a_{n}(x)\right)$. (We denote a meromorphic map by a dotted arrow). The meromorphic map $f(v)$ factors through $U^{\text {an }} \subset \mathbf{C}^{n}$ and hence we get a meromorphic map $F(v): D \cdots \rightarrow$ $U^{\text {an }} \subset V^{\text {an }}$. ( $U^{\text {an }}$ denotes the analytic space associated with the algebraic variety $U$. (For the definition see [S], Chap. $V$.) The meromorphic map $F(v)$ is defined independently of the choice of affine neighbourhood of the point $v$. Conversely we can recover the point $v$ from the meromorphic map $F(v)$.

Definition (1.1). We call the meromorphic map $F(v): D \cdots \rightarrow V^{\text {an }}$ the meromorphic map associated with the $L$-valued point $v: \operatorname{Spec} L \rightarrow V$.

Remark (1.2). Let $D$ be a domain of $\mathbf{C}$ and $L$ a differential field of meromorphic functions over $D$ with derivation $d / d x$. Let $C$ be a subfield of $L$ consisting of constants and $G$ an (absolutely) irreducible algebraic group defined over $C$. Let 
$v: \operatorname{Spec} L \rightarrow G_{L}$ be an $L$-rational point and $U$ an affine open neighbourhood of the image point $v^{\prime}: \operatorname{Spec} L \rightarrow G_{L} \rightarrow G$. The point $v^{\prime}$ is determined by a morphism $C[U] \rightarrow L$ of $C$-algebras. If $C[U]=C\left[u_{1}, u_{2}, \ldots, u_{n}\right]$ and if the morphism $C[U] \rightarrow L$ is defined by $u_{i} \rightarrow a_{i} \in L(1 \leq i \leq n), v$ defines a meromorphic map $F(v): D \cdots \rightarrow\left(U \otimes_{C} \mathbf{C}\right)^{\text {an }}, x \rightarrow\left(a_{1}(x), a_{2}(x), \ldots, a_{n}(x)\right)$ associated with the $L$-valued point $v$. Replacing $D$ by an open set if necessary, we may assume that $F(v)$ is regular on $D$. Then the analytic curve $F=F(v): D \rightarrow\left(G \otimes_{C} \mathbf{C}\right)^{\text {an }}$ defines a vector field $X_{F}$ along it:

$$
X_{F(\xi)}(f)=\lim _{h \rightarrow 0} \frac{f(F(\xi+h))-f(\xi)}{h} \text { for } f \in \mathscr{O}_{G_{\mathrm{C}} F(\xi)} .
$$

By right translation $R_{F(\xi)^{-1}}$, we get a map $D \rightarrow T_{G_{\mathrm{C}^{1}}}, \xi \rightarrow R_{F(\xi)^{-1}} X_{F(\xi)}$ which can be identified with $l \delta(v)$ (cf. [U2], p. 784 and [U4], Part II, §2). Here $T_{G_{\mathrm{C}^{1}}}$ denotes the tangent space at $1 \in G_{\mathbf{C}}$.

We give some examples to illustrate the idea.

Examples (1.3). Let us take as $G$ the additive group $\mathbf{G}_{a c}=\operatorname{Spec} C[t]$ and let $v: \operatorname{Spec} L \rightarrow \mathbf{G}_{a L}=\operatorname{Spec} L[t]$ be an $L$-rational point given by $L[t] \rightarrow L, t \rightarrow$ $\tau \in L$. For the definition of the additive group see (A5). Then $\delta(v)(t)=$ $\delta\left(v^{*}(t)\right)=\delta(\tau)$. Hence $\delta(v)=\delta(\tau)(d / d t)_{t=\tau}$ and $l \delta(v)=\delta(\tau)(d / d t)$ since $d / d t$ is (right) invariant.

(1.4) Let us take as $G$ the multicative group $\mathbf{G}_{m c}=\operatorname{Spec} C\left[t, t^{-1}\right]$ and let $v: \operatorname{Spec} L \rightarrow \mathbf{G}_{m L}=\operatorname{Spec} L\left[t, t^{-1}\right]$ be an $L$-rational point given by $L\left[t, t^{-1}\right] \rightarrow$ $L, t \rightarrow \tau \in L \quad\left(\mathrm{cf}\right.$. (A5)). Then $\delta(v)(t)=\delta\left(v^{*}(\tau)\right)=\delta(\tau)$. Hence $\delta(v)=\delta(\tau)$ $(d / d t)_{t=\tau}$ considered as a tangent vector at $\tau$ and

$$
l \delta(v)=\frac{\delta(\tau)}{\tau}\left(t \frac{d}{d t}\right)
$$

since $t \frac{d}{d t}$ is (right) invariant and hence in Lie $\mathbf{G}_{m c}$.

(1.5) Let us take as $G$ the general linear group $\mathbf{G L}_{n c}=\operatorname{Spec} C\left[t_{i j}\right.$, $\left.1 / \operatorname{det}\left(t_{i, j}\right)\right]_{1 \leq i, j \leq n}$ and let $v: \operatorname{Spec} L \rightarrow \mathbf{G L}_{n L}=\operatorname{Spec} L\left[t_{i j}, 1 / \operatorname{det}\left(t_{i j}\right)\right]_{1 \leq i, j \leq n}$ be an $L$-rational point given by $t_{i j} \rightarrow a_{i j}$. Then $\delta(v)=\sum_{1 \leq i, j \leq n} a_{i j}^{\prime} \frac{\partial}{\partial t_{i j}}$ or $l \delta(v)=$ $\left(a_{i j}^{\prime}\right)\left(a_{i j}\right)^{-1} \in \mathrm{gl}_{n}(L)$ as we have seen in [U4]. 
The following definition of $G$-primitive extension was introduced by Kolchin.

Definition (1.6). Let $L$ be a differential field and $K$ a differential subfield of $L$. Let $C$ be a subfield of $K$ consisting of constants and let $G$ be an algebraic group defined over $C$. Let $v: \operatorname{Spec} L \rightarrow \mathbf{G}_{L}$ be an $L$-rational point such that $l \delta(v) \in$ Lie $G_{L}=$ Lie $G \otimes_{C} L$ lies in Lie $G_{K}=$ Lie $G \otimes_{C} K$. Let $v^{\prime}:$ Spec $L \rightarrow G_{K}$ be the corresponding $L$-valued point ( $v^{\prime}$ is a composite of $v: \operatorname{Spec} L \rightarrow G_{L}$ and the canonical morphism $\left.G_{L} \rightarrow G_{K}\right)$. Then the quotient field $N$ of the image $\mathscr{O}_{G_{R} v^{\prime}} \rightarrow L$ is a differential field extension of $K$ and is finitely generated over $K$ as an abstract field extension (cf. [K] and [U2]). This extension $N / K$ is called a $G$-primitive extension of $K$.

Here are examples of $G$-primitive extensions. It is easy to see that the permissive operation (P5) is equivalent to allowing $G$-primitive extension such that the algebraic group $G$ is the abelian variety $\mathbf{C}^{n} / \Gamma$.

Examples (1.7). (1) Let $L$ be a differential field and $K$ be a differential subfield of $L$. The differential field $K$ contains the field $\mathbf{Q}$ of rational numbers and the multiplicative group $\mathbf{G}_{m}$ is defined over $\mathbf{Q}$. If $L=K(a)$ and $a^{\prime} \in K$, then $L / K$ is a $\mathbf{G}_{\boldsymbol{a}}$-primitive extension by Example (1.3).

(2) Let $L$ be a differential field and $K$ a differential subfield of $L$. If $L=$ $K(a)$ with $a^{\prime} / a \in K$, then $L / K$ is $\mathbf{G}_{m}$-primitive by Example (1.4).

(3) Let $L$ be a differential field and $K$ a differential subfield of $L$. If $L=$ $K\left(a_{i j}\right)_{1 \leq i, j \leq n}$ with $\operatorname{det}\left(a_{i j}\right) \neq 0$ and if $\left(a_{i j}^{\prime}\right)\left(a_{i j}\right)^{-1} \in \mathrm{gl}_{n}(K)$, then $L / K$ is a $\mathbf{G L}_{n}$-primitive extension by Example (1.5).

Let us introduce a group theoretic construction of new functions.

Definition (1.8). Let $K$ be a differential field of known functions (by which we mean given functions) meromorphic over a domain $D \subset \mathbf{C}$. We assume that the field $K$ contains the field $\mathbf{C}$ of constant functions. Let $L \supset K$ be another differential field of meromorphic functions over $D$. If the extension $L / K$ is $G$-primitive for an algebraic group $G$ defined over field $\mathbf{C}$, then we say that $L$ is a differential field of the newly known functions (which mean newly obtained functions) by operation $(\mathrm{Q})$.

The following theorem is very important and has applications for the sovability of differential equations (cf. [U2], [U3] and [U4]). In particular it contains a 
classical theorem of Poincaré: an algebraic differential equation of first order, free from movable critical points is solvable by permissible operations (0), (P1) (P2), ..,(P5)

THEOREM (1.9). A finite iteration of the permissible operations (0), (P1) (P2), .., (P5) is equivalent to a finite iteration of the operation (Q) (see [U4]).

The theorem shows that the permissible operations are group theoretic in nature. It is natural to adopt the following definition.

Definition (1.10). A meromorphic function $f$ over a domain $D \subset \mathbf{C}$ is classical if the function $f$ is obtained from the field $\mathbf{C}(x)$ of the rational functions of one variable by a finite iteration of permissible operations $(\mathrm{O}),(\mathrm{P} 1)(\mathrm{P} 2), \ldots$, (P5).

So far we worked over a differential field of meromorphic functions on a domain $D \subset \mathbf{C}$ with derivation $d / d x$. More generally we may replace the domain $D$ by a Riemann surface $p: R \rightarrow \mathbf{P}_{\mathbf{C}}^{1}$. We are interested in functions on a Riemann surface over $\mathbf{P}_{\mathbf{C}}^{1}$. Let us make the definition precise.

Definition (1.11). (i) A Riemann surface is a (connected and Hausdorff) complex manifold of dimension 1 .

(ii) A subset $U$ of a Riemann surface $S$ is a Zariski open set if the complement $U^{C}$ is a closed analytic subset of $S$.

(iii) Let $p: R \rightarrow S$ be a morphism of Riemann surfaces. We say that $p$ is an almost covering morphism or $R$ is an almost covering (without making $p$ precise) if there exist morphisms $p_{i}: R_{i} \rightarrow R_{i+1}, 0 \leq i \leq n-1$, of Riemann surfaces such that $p_{i}\left(R_{i}\right)$ is a Zariski open set of $R_{i+1}$ for all $0 \leq i \leq n-1, p=p_{n-1} \circ \cdots \circ p_{1}$ 。 $p_{0}$ and such that $R=R_{0}$ and $R_{n}=S$.

(iv) The morphism $p: R \rightarrow S$ or Riemann surface $R$ is called locally a covering if the image $p(R)$ does not reduce to a point.

It is known that a Riemann surface satisfies the second countability axiom (cf. [W]).

A subset $U$ of a Riemann surface $S$ is a Zariski open set if and only if one of the following condition is satisfied (i) $U=\phi$, (ii) the complement $F=U^{c}$ is locally finite, namely there exists an open covering $S=\cup_{i \in I} V_{\imath}$ such that $V_{i} \cap F$ is a finite (or empty) set any $i \in I$. 
As we proved in [U4], the permissible operations are reduced to the two operations; (P4) solution of linear differential equations, (P5) substitution of known functions into abelian functions. Since the essential singular points of a solution of a linear differential equation with coefficients meromorphic on a domain $\tilde{D} \subset \mathbf{C}$, are included in the set of poles of the coefficients, the set of essential singular points arising from the operation (P4) is a closed analytic subset of $D$. Similarly for the substitution of known functions into abelian functions, essential singularities of a newly known function by the operation (P5) form a closed analytic subset. Therefore for a classical function $f$ meromorphic on a domain $D$, by analytic continuation we can find an almost covering $p: R \rightarrow \mathbf{P}_{\mathbf{C}}^{1}$ such that $f$ is meromorphic on $R$. This is proved by induction on the number of times using the permissible operations to obtain the function $f$.

We consider the projective line $\mathbf{P}_{\mathbf{C}}^{1}$ as a union of $\operatorname{Spec} \mathbf{C}[x]$ and the point at infinity, which we denote by $\infty$. We identify the analytic manifold (Spec $\mathbf{C}[x])^{\text {an }}$ associated with $\operatorname{Spec} \mathbf{C}[x]$ with the complex plane $\mathbf{C}$. We have a rational vector field $d / d x$ on the projective line regular over Spec $\mathbf{C}[x]$. Therefore if we consider a field of classical functions meromorphic over a domain and finitely generated over $\mathbf{C}(x)$, we can find an almost covering $p: R \rightarrow \mathbf{P}_{\mathbf{C}}^{1}$ such that the field $K$ is a field of meromorphic functions over $R$. When the number of the meromorphic function is equal to one, this fact follows from the above assertion. The general case is proved by the same method.

Let $p: R \rightarrow \mathbf{P}_{\mathbf{C}}^{1}$ be locally a covering morphism of Riemann surfaces. Let $L$ be a field of meromorphic functions on $R$ closed under the derivation $d / d x$. We assume that $L \supset \mathbf{C}(x)$. Then we can speak of successive $G$-primitive extension of $\mathbf{C}(x)$ in the differential field $L$ and we can define classical functions meromorphic over $R$.

Let $r: R \rightarrow \mathbf{P}_{\mathbf{C}}^{1}, s: S \rightarrow \mathbf{P}_{\mathbf{C}}^{1}$ and $p: R \rightarrow S$ be locally a covering morphism of Riemann surfaces with $r=s^{\circ} p$ and let $f$ be a meromorphic function over $S$. Since $p^{*}: K(S) \rightarrow K(R) \quad\left(p^{*} g=p^{\circ} g\right.$ for $\left.g \in K(S)\right)$ is an (injective) morphism of fields and since the derivation $d / d x$ is fixed, the function $f$ is classical if and only if $p^{*} f$ is classical. Here $K(X)$ denotes the field of meromorphic functions over a Riemann surface $X$.

Let $U, V \subset \mathbf{C}$ be domains and $f, g$ classical functions meromorphic respectively on $U$ and $V$. Replacing $U$ and $V$ by subdomains, we may assume that there exist an almost covering morphism $p: R \rightarrow \mathbf{P}_{\mathbf{C}}^{1}$, holomorphic maps $r: U \rightarrow R, s:$ $V \rightarrow R$ with $p \circ r=\operatorname{Id}_{U}, p^{\circ} s=\operatorname{Id}_{V}$, meromorphic functions $\tilde{f}, \tilde{g}$ such that $\tilde{f} \circ r=f$, $\tilde{g} \circ s=g$. Hence we may define the sum $f+g$ and the product $f g$ using $\tilde{f}$ and $\tilde{g}$. But the following easy example shows that these are not well-defined. Let $U=V$ 
$=\mathbf{C}-\{a \in \boldsymbol{R} \mid a \geq 0\}$ and $f=g$ be a branch of $\log x$ with $\log 1=0$. Let $p: \mathbf{C}$ $\rightarrow \mathbf{C}^{*}$ be $u \rightarrow \exp u=x$. Let us define sections $s: U \rightarrow \mathbf{C}$ by $r(x)=u$ and $s: V$ $\rightarrow \mathbf{C}$ by $s(x)=u+2 \pi i$. If we take $\tilde{f}=u$ and $\tilde{g}=u-2 \pi i$, then $\tilde{f}+\tilde{g}=2 u-$ $2 \pi i$ which should be $2 u$.

The following theorem is proved in [U3].

THEOREM (1.12). If $f$ and $g$ are classical functions, then the composite function $g \circ f$ is classical.

In the assertion of Theorem (1.12) the definition of the composite function should be clarified. Let $U, V \subset C$ be domains such that the function $f$ is regular on $U, f(U) \subset V$ and such that the function $g$ is regular on $V$. Then we define $g \circ f$ as a composite $U \stackrel{f}{\rightarrow} V \stackrel{g}{\rightarrow} \mathbf{C}$.

Remark (1.13). At each step of construction of classical functions, the field of so far defined classical functions is finitely generated over $\mathbf{C}(x)$. Therefore if a finite set $S$ of classical functions meromorphic over locally a covering morphism are given, we can find a differential field $K$ which contains the set $S$ and is generated over $\mathbf{C}(x)$ as an abstract field.

We shall be concerned with finite sets, rings and fields of classical functions finitely generated over a subring of $\mathbf{C}(x)$ meromorphic over a Riemann surface. Therefore we may assume that there exists an almost covering $p: R \rightarrow \mathbf{P}_{\mathbf{C}}^{1}$ such that these functions are meromorphic over $R$. We recall that the derivation of a function field is always $d / d x$. We generalize Definition (1.1) by replacing the domain by a Riemann surface.

\section{§2. Q-classical functions}

Our idea of defining $\mathbf{Q}$-classical function is to start from $\mathbf{Q}(x)$ and at each step of the application of the operation $(\mathbf{Q})$, we assume that the algebraic group $G$ is defined over a small field of the known constant functions (e.g. in the first step, over $\mathbf{Q}$ ) and we admit as newly known functions only those satisfying the rationality conditions (or those whose initial condition on $G_{\mathbf{C}}$ at a rational point of a Riemann surface is rational). To this end we have to introduce the notion of rational points of a Riemann surface. 
Definition (2.1). Let $\boldsymbol{p}: R \rightarrow \mathbf{P}_{\mathbf{C}}^{1}$ be locally a covering morphism of Riemann surfaces and $K$ a field of meromorphic functions over $R$ which contains $\mathbf{Q}(x)$. Namely we have $f \circ p \in K$ for any rational function $f \in \mathbf{Q}(x)$ on $\mathbf{P}_{\mathbf{C}}^{1}$ with coefficients in $\mathbf{Q}$. The pair $\left(p: R \rightarrow \mathbf{P}_{\mathbf{C}}^{1}, K\right)$ will be denoted simply by $(R, K)$. For a point $P$ of $R$, we denote by $\mathscr{O}_{P}(K)$ a local ring $\{f \in K \mid f$ is regular at $P\}$ and by $m_{P}(K)$ the maximal ideal $\left\{f \in \mathscr{O}_{P}(K) \mid f(P)=0\right\}$ of the local ring. The residue field is naturally regarded as a subfield of $\mathbf{C}$ by taking the values of functions at $P$. Let $F$ be a subfield of $\mathbf{C}$. We say that a point $P$ of $R$ is $F$-valued point of $(R, K)$ if $f(P) \in F$ for every $f \in \mathscr{O}_{P}(K)$.

The notion of $F$-valued point is a natural generalization of the similar definition in algebraic geometry.

Example (2.2). A point $\left(x_{0}, x_{1}\right)$ of $\left(\mathbf{P}^{1}, \mathbf{Q}(x)\right)$ is $\mathbf{Q}$-valued if and only if $x_{0}=0$ or $x_{1} / x_{0}$ lies in $\mathbf{Q}$.

The following Lemma is useful in the sequel.

Lemma (2.3). Let $p: R \rightarrow \mathbf{P}_{\mathbf{C}}^{1}$ be locally a covering morphism. Let $K \supset \mathbf{Q}(x)$ be a field of meromorphic functions on $R$. Let $P$ be a point of $R$ such that the morphism $p$ is not ramified at $P$. Let $C$ be a subfield of $K$ consisting of the constants. Then the following conditions are equivalent.

(i) The point $P$ is a $C$-valued point of $(R, K)$.

(ii) Every Laurent coefficient of any function in $K$ at the point $P$ with respect to a local parameter $p^{*}(x-a)$ is in $C$ (If $a$ is the point at infinity, we understand $x-a$ to mean $1 / x)$.

Proof. As the case where $a$ is the point at infinity can be treated similarly, we may assume $a \in \mathbf{C}$. Since the question is local, we may further assume that $R$ is a domain of $\mathbf{C}$ and $p$ is the inclusion $R \subset \mathbf{C}$. The condition (ii) implies the condition (i) since in the condition (i) only the Laurent (Taylor) coefficients of constant terms of regular functions at $P$ are involved.

Conversely let us assume that the condition (i) is satisfied. If an element $f$ of $K$ has a Laurent expansion

$$
f=\sum_{n=m}^{\infty} a_{n}(x-a)^{n} \quad \text { with } \quad a_{m} \neq 0 .
$$

We show $a_{n} \in C$ for $n \geq m$ by induction. Since $x \in \mathscr{O}_{P}(K)$, the value $a$ of the 
function $x$ at $a$ is in $C$ and hence in $K$. Therefore $x-a \in K$ and hence $f(x)$ $(x-a)^{-m}=a_{m}+a_{m+1}(x-a)+\cdots \in K$. It follows from condition (i) that $a_{m}$ $\in C$. Assume now that $a_{j} \in C$ for $m \leq j \leq k-1$. Then $\sum_{n=m}^{k-1} a_{n}(x-a)^{n}$ is in $K$. Hence $\left\{f-\sum_{\jmath \gg-\infty}^{k-1} a,(x-a)^{j}\right\} /(x-a)^{k}=a_{k}+a_{k+1}(x-a)+\cdots$ is in $K$ and hence $a_{k} \in C$ by condition (i).

We adopt the following inductive definition. The definition will be illustrated by Examples (2.12), (2.13), (2.14) and (2.15).

Definition (2.4). The field of $\mathbf{Q}$-classical functions are generated inductively by the following rules.

(1) Let $p: R \rightarrow \mathbf{P}_{\mathbf{C}}^{1}, q: S \rightarrow R$ be locally covering morphisms of Riemann surfaces and let $f$ be a meromorphic function on $R$. Then $f$ is $\mathbf{Q}$-classical if and only if $f \circ q$ is $\mathbf{Q}$-classical.

(2) We start from the field $\mathbf{Q}(x)$ of meromorphic functions over $\mathbf{P}_{\mathbf{C}}^{1}$. Namely any rational function with coefficients in $\mathbf{Q}$ over $\mathbf{P}_{\mathbf{C}}^{1}$ (or any element of $\mathbf{Q}(x)$ ) is Q-classical.

(3) At each induction step, the inductively enlarged set $K$ of $\mathbf{Q}$-classical functions is a field of classical functions finitely generated over $\mathbf{Q}(x)$ and closed under the derivation $d / d x$. Hence there exists an almost covering $p: R \rightarrow \mathbf{P}^{1}$ such that $K$ is a field of meromorphic functions on $R$ as we observed at the end of $\S 1$ (notice that the field of constants of $K$ is also finitely generate over $\mathbf{Q}$ by [K] Chap. II, 11, Corollary 1.)

(4) Let $K$ be the differential field of so far defined $\mathbf{Q}$-classical functions. It follows from (3) that there exists locally a covering morphism $p: R \rightarrow \mathbf{P}_{\mathbf{C}}^{1}$ such that the field $K$ is a field of meromorphic functions on $R$. Let $\mathscr{K}$ be a differential subfield of $K$ containing $\mathbf{Q}(x)$, and $G$ an algebraic group defined over the field $C=C_{\mathscr{K}}$ of constants of $\mathscr{K}$. We allow a $G$-primitive extension of $K$ rational with respect to $\mathscr{K}$ in the following sense. Let $q: S \rightarrow R$ be locally a covering morphism of Riemann surfaces. Let $L$ be a differential field of meromorphic functions over $S$ with derivation $d / d x$ such that the field $K$ is identified with a differential subfield of $L$ by $q^{*}$. Let $v: \operatorname{Spec} L \rightarrow G_{L}$ be an $L$-rational point of $G_{L}$ satisfying the following conditions (A) and (B).

(A) There exists a Riemann surface $S^{\prime}$ and a point $P$ of $S^{\prime}$ such that

(1) $S$ is an open subsurface of $S^{\prime}$,

(2) $p^{\circ} q: S \rightarrow R \rightarrow \mathbf{P}_{\mathbf{C}}^{1}$ is extended over $S^{\prime}: r: S^{\prime} \rightarrow \mathbf{P}_{\mathbf{C}}^{1}$,

(3) the extended morphism $r$ is not ramified at the point $P$,

(4) any element of $\mathscr{K}$ is meromorphic over $S^{\prime}$, 
(5) the point $P$ is a $C$-rational point of $\left(S^{\prime}, \mathscr{K}\right)$,

(6) the meromorphic map $F(v): S^{\prime} \cdots \rightarrow G_{\mathbf{C}}$ associated with the $L$-valued point $v$ is regular at $P$ (cf. Definition (1.1)),

and such that

(7) the point $F(P) \in G_{C}$ comes from a $C$-rational point of $G$.

(B) $l \delta(v)$ which is an element of Lie $G \otimes L$ lies in Lie $G \otimes_{C} \mathscr{K}$.

Then $M=K\left(\left\{f \circ F(v) \mid f \in K\left(G_{K}\right)\right\}\right)=K(\{f \circ F(v) \mid f \in C(G)\})$ which is a differential subfield of $L$ is a newly defined $\mathbf{Q}$-classical functions meromorphic over $S$.

Remarks (2.5). The necessity of condition (A.3) will be discussed in $\$ 7$.

In (4) of Definition (2.4), at each step of the inductive construction, the field $K$ of so far defined $\mathbf{Q}$-classical functions is finitely generated over $\mathbf{Q}(x)$ as an abstract field. Hence the subfield $\mathscr{K}$ is finitely generated over $\mathbf{Q}(x)$ too.

It follows from the definition that the set of $\mathbf{Q}$-classical functions is a subfield of the field classical functions. Let $S$ be a finite set of $\mathbf{Q}$-classical functions. Then there exists a differential field of $\mathbf{Q}$-classical functions which contains the set $S$ and is finitely generated over $\mathbf{Q}$.

The logarithmic derivative $l \delta(v) \in$ Lie $G \otimes_{C} L$ lies in Lie $G \otimes_{C} \mathscr{O}_{P}(\mathscr{K})$ by conditions (A.5) and (B) of Definition (2.4). In fact the $L$-rational point $v$ :Spec $L \rightarrow \mathbf{G}_{L}$ arises from an $\mathscr{O}_{P}(L)$-valued point $\tilde{v}: \operatorname{Spec} \mathscr{O}_{P}(L) \rightarrow \mathbf{G}_{L}$ by condition (A.5). The point $\tilde{v}$ defines the $\mathscr{O}_{P}(L)$-valued point $\bar{v}: \operatorname{Spec} \mathscr{O}_{P}(L) \rightarrow \mathbf{G}_{\mathscr{O}_{P}(L)}$. Since $\mathscr{O}_{P}(L)$ is closed under $d / d x$, we get a tangent vector $\delta \tilde{v}$ on $\mathbf{G}_{\mathscr{O}_{P}(L)}$ at $\bar{v}$ by the argument of the definition of $l \delta(v)$. Therefore we can find a right invariant vector field $l \delta \bar{v} \in$ Lie $G \otimes_{C} \mathscr{O}_{P}(L)$. Since the construction is functorial, $l \delta(v)$ coincides with $l \delta(\bar{v})$ in Lie $G \otimes_{C} L$. Namely $l \delta(v) \in$ Lie $G \otimes_{C} \mathscr{O}_{P}(L)$. We conclude $l \delta(v) \in$ Lie $G \otimes_{C} \mathscr{O}_{P}(\mathscr{K})$ since $\mathscr{O}_{P}(L) \cap \mathscr{K}=\mathscr{O}_{P}(\mathscr{K})$.

If $r(P)$ coincides with the point at infinity of $\mathbf{P}_{\mathbf{C}}^{1}$, we can moreover show that $l \delta(v) \in$ Lie $G \otimes_{c} z^{2} \mathscr{O}_{P}(\mathscr{K})$, where $z=x^{-1}$ (we notice $z \in m_{P}(\mathcal{K})$ ). In fact we have $-z^{2} \frac{d}{d z}=\frac{d}{d x}$ and hence the image of $\delta(\bar{v}): \mathscr{O}_{P}(L) \rightarrow \mathfrak{O}_{P}(L)$ is contained in $z^{2} \mathscr{O}_{P}(L)$. Let $D_{1}, D_{2}, \ldots, D_{d}$ be a basis of the $C$-vector space Lie $G$. Let

$$
l \delta(v)=\sum_{\imath=1}^{d} g_{\imath} D_{\imath} \quad \text { with } \quad g_{\imath} \in \mathscr{O}_{P}(\mathscr{K})
$$

and $u_{1}, u_{2}, \ldots, u_{d}$ a regular system of parameters at the point $P$ of $G(F(P)$ is a $C$-rational point). Thus we have $\operatorname{det}\left(D_{i}\left(U_{j}\right)\right)_{1 \leq i, j \leq d}$ does not vanish at $F(P)$. Namely 


$$
\operatorname{det}\left(D_{i}\left(U_{j}\right)\right) \circ F(v) \in \mathscr{O}_{P}(L) \text { is a unit in } \mathscr{O}_{P}(L) .
$$

Operating (2.5.1) to $u_{j} \in C\left(G_{C}\right) \subset L\left(G_{L}\right)$, we conclude

$$
\sum_{i=1}^{d} g_{i} D_{i}\left(u_{j}\right)=l \delta(v)\left(u_{j}\right)
$$

which is an equality of rational functions on $G_{L}$. Taking $F(v)^{*}$ or considering the values of rational functions on $G_{L}$ of (2.5.3) at the $L$-rational point $v$, we get

$$
\begin{aligned}
\sum_{i=1}^{d} g_{i} D_{i}\left(u_{j}\right)(v) & =\delta\left(u_{j} \circ F(v)\right)=\frac{d}{d x}\left(u_{j} \circ F(v)\right) \\
& =-z^{2} \frac{d}{d z}\left(u_{j} \circ F(v)\right) \in z^{2} \mathscr{O}_{P}(L) .
\end{aligned}
$$

It follows from (2.5.2) and (2.5.4) $g_{i} \in z^{2} \mathscr{O}_{P}(L) \cap \mathscr{O}_{P}(\mathscr{K})=z^{2} \mathscr{O}_{P}(\mathscr{K})$.

Lemma (2.6). Using the notation in (4) of Definition (2.4) if we put $\mathcal{M}=\mathscr{K}(\{f$ 。 $\left.\left.F(v) \mid f \in \mathscr{K}\left(G_{\mathscr{K}}\right)\right\}\right)=\mathscr{K}(\{f \circ F(v) \mid f \in C(G)\}$, then the point $P$ is a $C$-valued point of $\left(S^{\prime}, \mathcal{M}\right)$.

Proof. In fact let $D_{1}, D_{2}, \ldots, D_{d}$ be a basis of the $C$-vector space of the right invariant derivations over $G_{C}$ and $D$ be a neighbourhood of the point $P$. First we assume that $r(P) \in \mathbf{C}, r$ being the morphism in (A2). Since the question is local and since the morphism $r$ is unramified at the point $P$, we may identify $D$ with a domain of $\mathbf{C}$. We may assume that there exists an affine open set $U=\operatorname{Spec} \mathbf{C}\left[u_{1}\right.$, $\left.u_{2}, \ldots, u_{n}\right]$ of $G$ such that the restriction $F(v) \mid D: D \rightarrow G_{\mathbf{C}}^{\text {an }}$ of the meromorphic map $F(v)$ associated with $v$ is regular and the image of $F(v)(D)$ is in $U_{\mathbf{C}}=U \otimes$ ${ }_{c}$ C. Let us denote the restriction $F(v) \mid D$ again by $F(v)$ so that we have $F(v): D$ $\rightarrow\left(U \otimes_{C} \mathbf{C}\right)^{\text {an }} \subset G_{\mathbf{C}}^{\text {an }}$. If we put $f_{i}=u_{i}{ }^{\circ} F(v)$, then $f_{i}$ is a holomorphic function on $D(1 \leq i \leq n)$ and the holomorphic $\operatorname{map} F(v): D \rightarrow\left(U \otimes_{C} \mathbf{C}\right)^{\text {an }}=\left(\operatorname{Spec} \mathbf{C}\left[u_{1}\right.\right.$, $\left.\left.u_{2}, \ldots, u_{n}\right]\right)^{\text {an }} \subset \mathbf{C}^{n}$ is defined by $x \rightarrow\left(f_{1}(x), f_{2}(x), \ldots, f_{n}(x)\right) \in \mathbf{C}^{n}$. Since $l \delta(v) \in$ Lie $G \otimes_{C} \mathscr{O}_{P}(\mathscr{K})$ as we noticed above, we have $l \delta(v)=\sum_{i=1}^{d} g_{i} D_{i}$ with $g_{i} \in \mathscr{O}_{P}(\mathcal{K})$. If we evaluate this equality at the point $v^{\prime}: \operatorname{Spec} L \rightarrow C_{\mathscr{K}}$ induced from the $L$-valued point $v: \operatorname{Spec} L \rightarrow G_{\mathscr{K}}$, we get

$$
\delta(v)=\sum_{i=1}^{d} g_{i} D_{i}(v)
$$

The latter is an equality in the vector space $\operatorname{Der}_{\mathscr{K}}\left(\mathscr{O}_{G_{\mathscr{K}} v^{\prime}}, L\right)$ of tangent vectors at $v^{\prime}$ on $G_{\mathscr{K}}$. If we apply the derivations in $(2.7)$ to $u_{j} \in C\left[u_{1}, u_{2}, \ldots, u_{d}\right] \subset \mathscr{O}_{G_{\mathscr{H}} v^{\prime}}$, we get 


$$
\delta(v)\left(u_{j}\right)=\sum_{i=1}^{d} g_{i} D_{i}(v)\left(u_{j}\right)
$$

Therefore

$$
f_{j}^{\prime}=\sum_{i=1}^{d} g_{i} D_{i}\left(u_{j}\right)(v)
$$

Since $D_{i}$ is defined over $C$ and regular on $U$, there exist polynomials $A_{i j}$ with coefficients in $C$ such that $D_{i}\left(u_{j}\right)=A_{i j}\left(u_{1}, u_{2}, \ldots, u_{n}\right)$ for $1 \leq i \leq d, 1 \leq j \leq n$. Hence

$$
f_{j}^{\prime}=\sum_{i=1}^{d} g_{i} \sum_{j} A_{\imath j}\left(f_{1}, f_{2}, \ldots, f_{n}\right)
$$

This shows $f_{j}^{\prime}(P) \in C$ for $1 \leq j \leq n$ since the $g_{i}$ 's and the $f_{i}$ 's are regular at $P$ and since their values at $P$ are in $C$. Differentiating (2.8), we get

$f_{j}^{\prime \prime}=$ polynomial in $g_{i}, g_{i}^{\prime}(1 \leq i \leq d)$ and $f_{k}, f_{k}^{\prime}(1 \leq 1 \leq k \leq n)$ with coefficients in $C$.

Using again (2.8), we get

$$
f_{j}^{\prime \prime}=\text { polynomial in } g_{i} \text { and } g_{i}^{\prime}(1 \leq i \leq d) \text { and } f_{k}(1 \leq k \leq n) .
$$

Hence by $(2.9) f_{j}^{\prime \prime}(P)$ is in $C$. Repeating this procedure, we conclude that the Taylor coefficients of the $f_{j}$ 's at $P$ with respect to the local parameter $x-P$ are all in $C$. Since the point $P$ is a $C$-rational point of $\left(S^{\prime}, \mathscr{K}\right)$, by Lemma (2.3) the Laurent coefficients of any element of $\mathscr{K}$ at $P$ with respect to the local parameter $x-P$ are in $C$. Since the $f$,'s $(1 \leq j \leq n)$ generate the field $\mathcal{M}$ over $\mathscr{K}$, for any function $f \in \mathscr{K}$ every Laurent coefficient of $f$ is in $C$. Therefore the point $P$ is a $C$-valued point of $\left(S^{\prime}, \mathcal{M}\right)$. If the point $r(P)$ coincides with the point at infinity of $\mathbf{P}_{\mathbf{C}}^{1}$, we can argue similarly using the fact that $l \delta(v) \in$ Lie $G \otimes_{C} z^{2} \mathscr{O}_{P}(\mathscr{K})$.

Definition (2.10). A $\mathbf{Q}$-classical function which is constant is called a classical number. The field of the classical numbers is denoted by $\mathbf{Q}^{\mathrm{cl}}$.

There are not too many $\mathbf{Q}$-classical functions as the following proposition shows.

Proposition (2.11). The field of $\mathbf{Q}$-classical functions consists of countable elements. In particular the field $\mathbf{Q}^{\mathrm{cl}}$ of the classical numbers is countable. 
Proof. In fact we prove at each step of inductive construction the choice is countable. We start from the field $\mathbf{Q}(x)$ consisting of countable elements. We choose an algebraic group $G$ defined over $\mathbf{Q}$. This choice is countable since the set $\mathbf{Q}$ is countable. We construct a $G$-primitive extension satisfying the rationality condition of Definition (2.4.4). The extension is determined by $l \delta(v) \in \operatorname{Lie} G \otimes_{\mathbf{Q}}$ $\mathbf{Q}(x)$ and the field $\mathbf{Q}(x)$ is countable. Therefore the choice of $l \delta(v)$ is countable. The differential equation for $v$ such that $l \delta(v)$ is equal to a given element in Lie $G$ $\otimes_{\mathbf{Q}} \mathbf{Q}(x)$ determines an almost covering $p: R \rightarrow \mathbf{P}_{\mathbf{C}}^{1}$ unramified over a Zariski open set of $\mathbf{P}_{\mathbf{C}}^{1}$. Among such covering we consider only those whose image $p(R)$ is maximum. Among those covering, there exists the universal one which we denote by $p: R \rightarrow \mathbf{P}_{\mathbf{C}}^{1}$ by abuse of notation. Since furthermore the choice of $\mathbf{Q}$-rational points $P$ on the Riemann surface $R$ and that of $\mathbf{Q}$-rational points on $G$ are countable. Therefore the field of $\mathbf{Q}$-classical functions obtained by the first step consists of countable elements. We can apply this argument for the further step of inductive construction if we observe the following.

(1) Since $\mathscr{K}=\mathbf{Q}(x)\langle f\rangle\left(=\mathbf{Q}\left(x, f, f^{\prime}, f^{(2)}, \ldots\right)\right)$ for a suitable $f \in \mathscr{K}$ by [K], Chap. II, 8, Proposition 9, the choice of the differential subfield $\mathscr{K}$ is countable.

(2) Since $r: S^{\prime} \rightarrow \mathbf{P}_{\mathbf{C}}^{1}$ is not ramified at $P$, we may assume that $S^{\prime}$ is a domain of $\mathbf{C}$. Hence there is no choice of $S^{\prime}$ but a choice of $C$-valued point $P$ of $\left(S^{\prime}, \mathcal{K}\right)$. In particular $P$ is a $C$-valued point of $\left(S^{\prime}, \mathbf{Q}(x)\right)$. The choice of $P$ as a $C$-valued point of $\left(S^{\prime}, \mathbf{Q}(x)\right)$ is countable. The extension of $C$-valued point of $\left(S^{\prime}, \mathbf{Q}(x)\right)$ to a $C$-valued of $\left(S^{\prime}, \mathscr{K}\right)$ is countable since the number of branches of a Riemann surface over which $\mathscr{K}$ is meromorphic is at most countable.

Now we give several examples to illustrate our idea. The most general form of Examples (2.12), (2.14), (2.15) is given by Theorem (3.7) in $\S 3$.

Example (2.12). Let us consider the first step in Definition (2.4) as follows. Since we start from $\mathbf{Q}(x)$, necessarily $K=\mathscr{K}=\mathbf{Q}(x), C=\mathbf{Q}$. We choose $R=S$ $=S^{\prime}=\mathbf{C} \subset \mathbf{P}_{\mathbf{C}}^{1}\left(p: \mathbf{C} \rightarrow \mathbf{P}_{\mathbf{C}}^{1}\right.$ being the inclusion). As an algebraic group $G$ we take $\mathbf{G}_{m} \mathbf{Q}=\operatorname{Spec} \mathbf{Q}\left[t, t^{-1}\right]$ and $L=\mathbf{Q}(x$, exp $x)$. We consider an $L$-valued point $v: \operatorname{Spec} L \rightarrow \mathbf{G}_{m L}=\operatorname{Spec} L\left[t, t^{-1}\right]$ defined by $t \rightarrow \exp x$. The associated meromorphic map, which is holomorphic in this case, $F(v): \mathbf{C} \rightarrow \mathbf{G}_{m}^{\text {an }} \mathbf{C}=\mathbf{C}^{*}=\mathbf{C}$ $-\{0\}$ is given by $x \rightarrow \exp x$. We take $P=0$ in (2.4.4.A) which is a $\mathbf{Q}$-rational point of $(\mathbf{C}, \mathbf{Q}(x))$. Then as we have shown in Example (1.3), $l \delta(v)=t \frac{d}{d t}$ hence $l \delta(v) \in$ Lie $G_{m} \mathbf{Q}$. Moreover at the $\mathbf{Q}$-rational point $P$ of $(\mathbf{C}, \mathbf{Q}(x)), F(v)=$ 
$\exp x: \mathbf{C} \rightarrow \mathbf{G}_{m}^{\text {an }} \mathbf{C}=\mathbf{C}^{*} \subset \mathbf{C}$ takes the value $1 \in \mathbf{Q}$ which is a $\mathbf{Q}$-rational point of $\mathbf{G}_{m} \mathbf{Q}$. Therefore we get a field of $\mathbf{Q}$-classical functions $K(t \circ \exp x)=$ $K(\exp x)=\mathbf{Q}(x, \exp x)$.

Now let us pass to the following second step. We take in Definition (2.4), $K=$ $\mathbf{Q}(x, \exp x), \mathscr{K}=\mathbf{Q}(x), L=\mathbf{Q}(x, \exp x, \exp (x+1))$ so that $C=C_{\mathscr{K}}=\mathbf{Q}$. Again we take $R=S=S^{\prime}=\mathbf{C} \subset \mathbf{P}_{\mathbf{C}}^{1}$. Let $v: L \rightarrow \mathbf{G}_{m L}$ be an $L$-rational point defined by $t \rightarrow \exp (x+1)$. The associated holomorphic map $F(v): \mathbf{C} \rightarrow \mathbf{G}_{m}^{\text {an }} \mathbf{C}=\mathbf{C}^{*} \subset \mathbf{C}$ is given by $x \rightarrow \exp (x+1)$. We take as a $\mathbf{Q}$-rational point of $(\mathbf{C}, \mathbf{Q}(x))$ $P=-1$. We see $l \delta(v)=t \frac{d}{d t}$ which in Lie $\mathbf{G}_{m \mathbf{Q}} \subset$ Lie $\mathbf{G}_{m \mathscr{K}}$. The point $P$ is a $C=\mathbf{Q}$-rational point of $(\mathbf{C}, \mathscr{K})$ and at this point the associated holomorphic map $F(v)=\exp (x+1): \mathbf{C} \rightarrow \mathbf{G}_{m}^{\text {an }} \mathbf{C}$ takes the value $1 \in \mathbf{G}_{m} \mathbf{C}$ which is a $\mathbf{Q}$-rational point of $\mathbf{G}_{m} \mathbf{C}$. Therefore $M=K(\exp (x+1))$ is a field of $\mathbf{Q}$-classical functions obtained in this second step. Since $\exp x$ is in $K$ and hence in $M, e=\exp (x+$ $1)(\exp x)^{-1}$ is a $\mathbf{Q}$-classical function and hence is a classical number. This argument shows that for any rational number $r \in \mathbf{Q}$, the number $e^{r}$ is classical.

Example (2.13). $\quad e^{2 \pi \imath / n}$ is a classical number for $n \in \mathbf{N}$ (see Theorem (3.1) in $\S 3)$, where $i=\sqrt{-1}$. Consequently any element of a cyclotomic field is classical.

In fact we consider the following first step. We take necessarily $K=\mathscr{K}=$ $\mathbf{Q}(x)$ and $C=C_{\mathscr{K}}=\mathbf{Q}$. As an algebraic group we choose $\mathbf{G}_{m} \mathbf{C}$. Consider a covering $p: R=\mathbf{C}^{*} \rightarrow \mathbf{C}^{*} \subset \mathbf{P}_{\mathbf{C}}^{1}$ defined by $z \rightarrow z^{n}=x$. We take $S=S^{\prime}=R$ and $L$, $=\mathbf{Q}(x, z \exp (-2 \pi i j / n))$ for $0 \leq j \leq n-1$. Since $d z / d x=z / n x, L$, is a differential field. Let $v_{j}: \operatorname{Spec} L_{j} \rightarrow \mathbf{G}_{m L_{j}}=\operatorname{Spec} L_{j}\left[t, t^{-1}\right]$ be an $L_{j}$-valued point defined by $t \rightarrow z \exp (-2 \pi i j / n)$. As for the point $P$ of Definition (2.4.4) (A.5), depending on $j$ we take $P,=\exp (2 \pi i j / n) \in\left(\mathbf{C}^{*}, p^{*} \mathbf{Q}(x)\right)$, which is a $\mathbf{Q}$-rational point of $\left(\mathbf{C}^{*}, p^{*} \mathbf{Q}(x)\right)$. By Example $(1.7) l \delta\left(v_{j}\right)=[d z \exp (-2 \pi i j / n) / d x] /$ $[z \exp (-2 \pi i j / n)] t \frac{d}{d t}=\frac{1}{z} \frac{d z}{d x} t \frac{d}{d t}=\frac{1}{n} t \frac{d}{d t} \in$ Lie $\mathbf{G}_{m} \mathbf{Q}$. The associated meromorphic map $F\left(v_{j}\right): \mathbf{C}^{*} \rightarrow \mathbf{G}_{m \mathbf{C}}^{\text {an }}, z \rightarrow z \exp (-2 \pi i j / n)$ takes at $P, \in\left(\mathbf{C}^{*}\right.$, $\left.p^{*} \mathbf{Q}(x)\right)$ the value $1 \in \mathbf{G}_{m} \mathbf{C}$ which is a $\mathbf{Q}$-rational point. Therefore $K(z \exp$ $(-2 \pi i j / n))$ is a field of $\mathbf{Q}$-classical functions. Arguing as in the preceding example, we conclude that $\exp (2 \pi i / n)=z \exp (-2 \pi i j / n) /[z \exp (-2 \pi i(j+1) /$ $n]$ is a $\mathbf{Q}$-classical function and hence a classical number.

EXAmple (2.14). The circular constant $\pi$ is a classical number. Let us consider the following first step of Definition (2.4). Necessarily $K=\mathscr{K}=\mathbf{Q}(x)$ so that 
$C=C_{\mathscr{K}}=\mathbf{Q}$. We take $G=\mathbf{G}_{a \mathbf{Q}}$. We choose $S=S^{\prime}=R=\mathbf{C}$ with morphism $p: \mathbf{C}=R \rightarrow \mathbf{C} \subset \mathbf{P}_{\mathbf{C}}^{1}, z \rightarrow \exp z=x$. Namely the Riemann surface $R$ is isomorphic to $\mathbf{C}$ but it covers $\mathbf{C} \subset \mathbf{P}_{\mathbf{C}}^{1}$. Let $L=\mathbf{Q}(x, z)$. The field $L$ is closed under the derivation $d / d x$ since $d z / d x=x^{-1}$. We choose an $L$-valued point $v: L \rightarrow \mathbf{G}_{a} \mathbf{C}$ $=\operatorname{Spec} L[t]$ defined by $t \rightarrow z$ so that the associated meromorphic map $F(v): R=$ $\mathbf{C} \rightarrow \mathbf{G}_{a \mathbf{C}}^{\text {an }}$ is defined by $z \rightarrow z$. We take as $P$ a $\mathbf{Q}$-rational point 0 of $\left(R, p^{*} \mathbf{Q}(x)\right)$. It follows from Example (2.13) that $l \delta(v)=\frac{d z}{d x} \frac{d}{d t}=\frac{1}{x} \frac{d}{d t} \in \operatorname{Lie} \mathbf{G}_{a \mathbf{Q}^{(x)}}$. At $P=0$ the map $F(v): R \rightarrow \mathbf{G}_{a \mathbf{C}}^{\text {an }}$ is regular and takes the value 0 which is a $\mathbf{Q}$-rational point of $\mathbf{G}_{a \mathbf{Q}} \cdot M=K(t \circ f(x))=K(z)$ is the field of $\mathbf{Q}$-classical functions obtained by the first step.

Now we pass to the second step. We take in Definition (2.4) $K=\mathbf{Q}(x, z), \mathscr{K}$ $=\mathbf{Q}(x)$ so that $C=C_{K}=\mathbf{Q}$. Let $S=S^{\prime}=R=\mathbf{C}$ be as above, $L=\mathbf{Q}(x, z, z$ $+2 \pi i), G=\mathbf{G}_{a \mathbf{Q}}$. Let $v: \operatorname{Spec} L \rightarrow \mathbf{G}_{a L}=\operatorname{Spec} L[t]$ be defined by $t \rightarrow z+$ $2 \pi i$. We take as $P$ a $\mathbf{Q}$-rational point $z=2 \pi i$ of $\left(R, p^{*} \mathbf{Q}(x)\right)$ so that the associated holomorphic map $F(v): R \rightarrow \mathbf{G}_{a \mathbf{C}}^{\text {an }}$ is defined by $z \rightarrow z+2 \pi i$. Then by Example (2.13) $l \delta(v)=\frac{1}{x} \frac{d}{d t} \in \operatorname{Lie} \mathbf{G}_{a} \otimes_{\mathbf{Q}} \mathscr{K}$. At the point $P$ the map $F(v): R \rightarrow$ $\mathbf{G}_{a \mathbf{C}}^{\text {an }}$ takes the value 0 which is a $\mathbf{Q}$-rational point of $\mathbf{G}_{a \mathbf{Q}}$. Therefore $M=K(z$ $+2 \pi i)=\boldsymbol{Q}(x, z, z+2 \pi i)$ is a field of $\mathbf{Q}$-classical functions obtained by the second step. Therefore $2 \pi i=z+2 \pi i-z$ is a classical number. Since $i$ is a classical number by Example (2.13), the circular constant $\pi$ is a classical number.

Remark (2.14.1). The argument of Example (2.14) shows that $\log x$, $\log (r x)=\log r+\log x$ and hence $\log r$ are $\mathbf{Q}$-classical for $r \in \mathbf{Q}$.

So far we treated the linear algebraic groups $\mathbf{G}_{a}$ and $\mathbf{G}_{m}$. The following example illustrates the case of elliptic curves.

Example (2.15). Let $G$ be an elliptic curve defined over $\mathbf{Q}$. Hence $G$ is isomorphic to a projective curve in $\mathbf{P}_{\mathbf{C}}^{2}$ defined by

$$
X_{0} X_{2}^{2}=4 X_{1}^{3}-g_{2} X_{0}^{2} X_{1}-g_{3} X_{0}^{3}, \quad g_{2}, g_{3} \in \mathbf{Q} .
$$

We take $(0,0,1)$ as the origin of the elliptic curve. In the affine form $G$ is defined by

$$
w^{2}=4 u^{3}-g_{2} u-g_{3}, \quad u=X_{1} / X_{0}, \quad w=X_{2} / X_{0} .
$$

We have $\mathbf{Q}(G)=\mathbf{Q}(u, w)$. Let $L$ be a lattice of $\mathbf{C}$ with $60 \sum_{\omega \in L^{\prime}} \omega^{-4}=g_{2}$ and 
$140 \sum_{\omega \in L^{\prime}} \omega^{-6}=g_{3}$ where $L^{\prime}=L-\{0\}$. Let

$$
\wp(x)=\wp(x ; L)=x^{-2}+\sum_{\omega \in L^{\prime}}\left\{(x-\omega)^{-2}-\omega^{-2}\right\}
$$

be the Weierstrass $\mathcal{P}_{\text {-function. Then }}$ the $\boldsymbol{\gamma}_{\text {function }}$ satisfies the differential equation

$$
\wp^{\prime 2}=4 \wp^{3}-g_{2} \wp-g_{3}
$$

so that we can define a holomorphic map

$$
\pi: \mathbf{C} \rightarrow\left(G \otimes_{\mathbf{Q}} \mathbf{C}\right)^{\text {an }}, x \rightarrow\left(1, \gamma^{\prime}(x), \gamma^{\prime}(x)\right) .
$$

A $\mathbf{Q}$-derivation $\delta: \mathbf{Q}(u) \rightarrow \mathbf{Q}(u, w), u \rightarrow w$ can be extended uniquely to a $\mathbf{Q}$-derivation which we denote by the same letter $\delta$. Using this convention we can show by a simple calculation that the $\mathbf{Q}$-derivation $\delta=v \frac{d}{d u}: \mathbf{Q}(u, w) \rightarrow \mathbf{Q}(u, w)$, which can be considered as a rational vector field on $G$, is everywhere regular on $G$. Since $G$ is complete, $G$ operates on $H^{0}(G, \Theta)$ trivially, where $\Theta$ is the tangent bundle of $G$. In particular the vector field $w \frac{d}{d u}$ is $G$-invariant. We consider a $\mathbf{Q}\left(\wp^{\wp}, \gamma^{\prime}\right)$-valued point $v^{\prime}: \operatorname{Spec} \mathbf{Q}\left(\boldsymbol{\gamma}^{\circ}, \boldsymbol{\gamma}^{\prime}\right) \rightarrow G$ defined by $u \rightarrow \wp, w \rightarrow \boldsymbol{\wp}^{\prime}$. For the corresponding $\mathbf{Q}\left({ }^{\circ}, \gamma^{\prime}\right)$-rational point $v *: \operatorname{Spec} \mathbf{Q}\left(\wp^{\prime}, \wp^{\prime}\right) \rightarrow G \otimes_{\mathbf{Q}} \mathbf{Q}\left({ }^{\circ}, \gamma^{\prime}\right)$, we get from the definition of $\delta(v), \delta(v)(u)=8^{\prime}$. Since $\left[\left(w \frac{d}{d u}\right)(u)\right]_{v}=w(v)=$ $\boldsymbol{\gamma}^{\prime}$, we have $l \delta(v)=w \frac{d}{d u} \in \operatorname{Lie} G \subset$ Lie $G_{\mathbf{Q}(x)}$. Since a $\mathbf{Q}$-rational point 0 of $(\mathbf{C}, \mathbf{Q}(x))$ is mapped to the origin $1 \in G_{\mathbf{C}}$ of the elliptic curve, $\wp(x ; L)=u \circ \pi$ and $\boldsymbol{\wp}^{\prime}(x ; L)=w^{\circ} \pi$ are $\mathbf{Q}$-classical functions if $g_{2}(L)$ and $g_{3}(L)$ are rational numbers. Now the argument of Examples (2.12) and (2.13) shows that $\gamma(x+r$ : $L)$ and $\boldsymbol{\gamma}^{\prime}(x+r: L)$ are $\mathbf{Q}$-classical functions for $r \in \mathbf{Q}$. On the other hand by the addition theorem of the $\gamma^{-}$-function, $\gamma^{\prime}(r)$ and $\gamma^{\prime}(r)$ are rationally expressed by $\boldsymbol{\gamma}^{\prime}(x), \boldsymbol{\gamma}^{\prime}(x), \boldsymbol{\gamma}^{\prime}(x+r)$ and $\boldsymbol{\gamma}^{\prime}(x+r)$ with coefficients in $\mathbf{Q}$. Hence $\boldsymbol{\gamma}(\boldsymbol{r}$ : $L)$ and $\boldsymbol{\gamma}^{\prime}(r ; L)$ are classical numbers for $r \in \mathbf{Q}$ if $g_{2}(L)$ and $g_{2}(L)$ are in $\mathbf{Q}$.

A natural generalization of Example (2.15) is as follows.

Example (2.16). Let $p: R \rightarrow \mathbf{P}_{\mathbf{C}}^{1}$ be locally a covering morphism of Riemann surfaces and $K \supset \mathbf{Q}(x)$ be a differential field of $\mathbf{Q}$-classical functions meromorphic over $R$. Let us assume that the field $K$ is finitely generated over $\mathbf{Q}(x)$. Let $C$ be the field of the constants of $K$. Hence the field $C$ consists of classical numbers. 
Let $A$ be an abelian variety defined over $C$ and $D_{1}, D_{2}, \ldots, D_{n} \in$ Lie $A=$ \{invariant vector field on $A$ defined over $C$ \} be a basis of the $C$-vector space Lie $A$. Then there exists a universal covering $\pi: C^{n} \rightarrow\left(A \otimes_{C} \mathbf{C}\right)^{\text {an }}$ such that $\pi_{*}\left(\partial / \partial x_{i}\right)=D_{i}$ for $1 \leq i \leq n$. Let $D \subset R$ be an open set which is mapped isomorphically onto a domain of $\mathbf{C} \subset \mathbf{P}_{\mathbf{C}}^{1}$. Let $\mathscr{F}: D \rightarrow \mathbf{C}^{n}$ be a holomorphic map sending $x$ to $\left(f_{1}(x), f_{2}(x), \ldots, f_{n}(x)\right)$. We put $F=\pi \circ \mathscr{F}$. Let us assume further that there exist a field $C_{1}$ of classical numbers finitely generated over $Q$ and a $C_{1}$-valued point $P$ of $(D, K)$ such that $\pi \circ F(P)$ is a $\mathbf{Q}^{\mathrm{cl}}$-valued point of $A$. Then for any $\varphi \in C(A), \varphi^{\circ} \pi^{\circ} F$ is $\mathbf{Q}$-classical. In fact, let us denote by $\mathbf{C}(D)$ the differential field of meromorphic functions on $D$ and let $v: \operatorname{Spec} \mathbf{C}(D) \rightarrow A_{\mathbf{C}(D)}$ be a $\mathbf{C}(D)$-valued point corresponding to the holomorphic map $\pi \circ F: D \rightarrow A_{\mathbf{C}}$ (cf. $\left.\S 1\right)$.

Since for $\varphi \in C(A) \subset K\left(A_{K}\right)$ we have

$$
\begin{gathered}
\delta(v)(\varphi)=\frac{d}{d x}\left(\varphi^{\circ} \pi^{\circ} \mathscr{F}\right)=\frac{d}{d x}\left(\varphi^{\circ} \pi\left(f_{1}(x), f_{2}(x), \ldots, f_{n}(x)\right)\right) \\
=\sum_{i=1}^{n} \frac{\partial}{\partial x_{i}}\left(\varphi^{\circ} \pi\right) \frac{d f_{i}}{d x}=\sum_{i=1}^{n} f_{i}^{\prime} \pi_{*}\left(\frac{\partial}{\partial_{x_{i}}}\right)(\varphi)=\left(\sum_{i=1}^{n} f_{i}^{\prime} D_{i}\right)(\varphi), \\
\delta(v)=\left.\sum_{i=1}^{n} f_{i}^{\prime} D_{i}\right|_{v}
\end{gathered}
$$

and hence $l \delta(v)=\sum_{i=1}^{n} f_{i}^{\prime} D_{i}$ lies in Lie $A_{K}$. Adding a finite number of classical numbers to $C_{1}$, we get a field $C_{2}$ of classical numbers finitely generated over $\mathbf{Q}$ such that the point $\pi \circ F(P) \in A_{C}$ comes from a $C_{2}$-valued point of $A_{C}$. Therefore if we put $\tilde{K}=K C_{2}$, then $\tilde{K}$ is a differential field of $\mathbf{Q}$-classical functions meromorphic over $D$, the field $\tilde{K}$ is finitely generated over $\mathbf{Q}(x)$, the point $P$ is a $C_{2}$-rational point of $(D, \tilde{K})$ by Lemma $(2.3)$ and $\pi \circ F(P)$ is a $C_{2}$-rational point. Therefore for any $\varphi \in C(A) \subset C_{2}\left(A_{C_{2}}\right), \varphi^{\circ} \pi \circ \mathscr{F}$ is $\mathbf{Q}$-classical by Definition (2.4).

\section{§3. Theorems on $\mathrm{Q}$-classical functions}

The following result generalizes Example (2.13).

Theorem (3.1). Any algebraic number is $\mathbf{Q}$-classical.

Proof. Let $a \in \mathbf{C}$ be an algebraic number and $F(t)=t^{n}+a_{1} t^{n-1}+\cdots+$ $a_{n} \in \mathbf{Q}[t]$ be the irreducible polynomial with $F(a)=0$. We may assume that $a$ is not a rational number. If we take a small circle $\mathscr{C}$ centered at $a$, then we have an integral representation

$$
a=\frac{1}{2 \pi i} \int_{\mathscr{C}} \frac{x F^{\prime}(x)}{F(x)} d x
$$


(see [D], Chap. VIII, §6 for example).

Let us consider a differential equation

$$
y^{\prime}-\frac{x F^{\prime}(x)}{2 \pi i F(x)}=0
$$

Let $H(x)$ be a solution of the differential equation $(*)$ and $p: \tilde{R} \rightarrow \mathbf{P}_{\mathbf{C}}^{1}$ be a Riemann surface over which $H(x)$ is singled valued. The map $p$ is not ramified over $1 \in \mathbf{C} \in \mathbf{P}_{\mathbf{C}}^{1}$ since $F(1) \neq 0$. We take a path joining 1 and $\mathscr{C}$ which does not encounter any root of $F(x)=0$. We get a loop $\mathscr{C}^{\prime}$ starting from 1 and coming back to 1 .

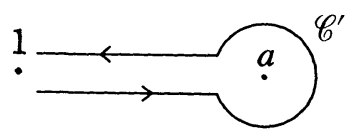

Let $P_{1}$ be a point of $\tilde{R}$ lying over 1 , the loop $\mathscr{C}^{\prime}$ is lifted over $\tilde{R}$ as a path joining $P_{1}$ and $P_{2}$ both lying over 1 . As we have $\left(H(x)-H\left(P_{1}\right)\right)-\left(H(x)-H\left(P_{2}\right)=\right.$ $H\left(P_{2}\right)-H\left(P_{1}\right)=\int_{\mathscr{C}^{\prime}} \frac{x F^{\prime}(x)}{2 \pi i F(x)} d x=\int_{\mathscr{C}} \frac{x F^{\prime}(x)}{2 \pi i F(x)} d x=a$, it is sufficient to show that $H(x)-H\left(P_{i}\right), i=1,2$ is a $\mathbf{Q}$-classical function. We prove that $H(x)-$ $H\left(P_{1}\right)$ is a $\mathbf{Q}$-classical function. For by the same method we can show that $H(x)-H\left(P_{2}\right)$ is $\mathbf{Q}$-classical.

It follows from Example (2.14) that there exists a differential field $\tilde{K}=$ $\mathbf{Q}(2 \pi i, x, \log x)$ of $\mathbf{Q}$-classical functions known by the two steps consisting of meromorphic functions on a small disc $\bar{R}$ centered at $1 \in \mathbf{C} \subset \mathbf{P}_{\mathbf{C}}^{1}$. Here we mean by $\log x$ a branch over $\bar{R}$ with $\log 1=0$. Let us consider a section $q: \bar{R} \rightarrow \tilde{R}$ such that $q(1)=P_{1}$. In Definition (2.4) we take $K=\tilde{K}=\mathbf{Q}(2 \pi i, x, \log x)$ and $\mathscr{K}=\mathbf{Q}(2 \pi i, x), L=K\left(q^{*} H\left(x-H\left(P_{1}\right)\right), R=\bar{R}=S=S^{\prime}\right.$. Then we have $C_{\mathscr{K}}$ $=\mathbf{Q}(2 \pi i)$ and $\frac{x F^{\prime}(x)}{2 \pi i x F(x)} \in \mathscr{K}$. We choose $G=\mathbf{G}_{a \mathbf{Q}(2 \pi i)}=\operatorname{Spec} \mathbf{Q}(2 \pi i)[t]$. Let us consider an $L$-rational point $v: L \rightarrow \mathbf{G}_{a L}=\operatorname{Spec} L[t]$ defined by $t \rightarrow H(x)-$ $H\left(P_{1}\right)\left(=q^{*} H(x)-H\left(P_{1}\right)\right)$. The associated holomorphic map $F(v): R \rightarrow \mathbf{G}_{a \mathbf{C}}^{\text {an }}$ is given by $x \rightarrow H(x)-H\left(P_{1}\right)$. As a $C_{\mathscr{K}}$-rational point $P$ of $(R, \mathscr{K})$ we take 1 . We have $l \delta(v)=\frac{d H(x)}{d x} \frac{d}{d t}=\frac{x F^{\prime}(x)}{2 \pi i F(x)} \frac{d}{d t} \in$ Lie $\mathbf{G}_{a} \otimes_{\mathbf{Q}^{(2 \pi i)}} \mathscr{K}$. At the $\mathbf{Q}(2 \pi i)$-rational point $P=1$ the associated holomorphic map $F(v): R \rightarrow \mathbf{G}_{a \mathbf{C}}^{\text {an }}$ takes the value $F(v)(1)=H\left(P_{1}\right)-H\left(P_{1}\right)=0$ which is a $\mathbf{Q}(2 \pi i)$-rational point of $\mathbf{G}_{a} \mathbf{Q}^{(2 \pi i)}$. Therefore $H(x)-H\left(P_{1}\right)=t \circ F(v)$ is a $\mathbf{Q}$-classical function. 
We can prove by the same argument

Corollary (3.2). Let $a_{1}, a_{2}, \ldots, a_{n}$ be classical numbers. If a complex number $b$ is algebraic over $\mathbf{Q}\left(a_{1}, a_{2}, \ldots, a_{n}\right)$, then $b$ is classical.

The following corollary is a cosequence of Corollary (3.2).

Corollary (3.3). The field $\mathbf{Q}^{\mathrm{cl}}$ of classical numbers is algebraically closed.

Lemma (3.4). Let $p: R \rightarrow \mathbf{P}_{\mathbf{C}}^{1}$ be locally a covering morphism of Riemann surfaces and $K \supset \mathbf{Q}(x)$ a differential field of meromorpic functions on $R$ with derivation $\frac{d}{d x}$. Let $C \subset K$ be a subfield of constants of $K$. If the field $K$ is generated by a finite number of elements $a_{1}, a_{2}, \ldots, a_{n}$ over $C$ as an abstract field, then there exists a non-empty Zariski open set $W$ such that if $Q \in W$, then (1) $p(Q)$ is not the point at infinity of $\mathbf{P}_{\mathbf{C}}^{1}$, (2) the morphism $p$ is not ramified at $Q$ so that $x-p(Q)$ is a local $p a$ rameter at $Q$, (3) the $a_{i}$ 's are regular at $\mathbf{Q}$ and we have (4) $\left\{f(Q) \mid f \in O_{Q}(K)\right\}=$ $C\left(a_{1}(Q), a_{2}(Q), \ldots, a_{n}(Q)\right)$.

Proof. We may assume that $R$ is a domain of $\mathbf{C}$ since the conditions (1) and (2) are satisfied on a non-empty Zariski open set. Since the field $K$ is closed under the derivation $d / d x$, there exist polynomials $A_{i}, B \in C\left[X_{1}, X_{2}, \ldots, X_{n}\right]$ such that

$$
d a_{i} / d x=A_{i}\left(a_{1}, a_{2}, \ldots, a_{n}\right) / B\left(a_{1}, a_{2}, \ldots, a_{n}\right)
$$

for $1 \leq i \leq n$. Let $W=\left(Q \in R \mid\right.$ the $a_{1}$ 's are regular at $Q$ and $B\left(a_{1}(Q), a_{2}(Q)\right.$, $\left.\left.\ldots, a_{n}(Q)\right) \neq 0\right\}$. Then if $Q \in W$, then it follows from (3.4.1),

$$
a_{i}^{\prime}(Q)=A_{i}\left(a_{1}(Q), a_{2}(Q), \ldots, a_{n}(Q)\right) / B\left(a_{1}(Q), a_{2}(Q), \ldots, a_{n}(Q)\right) .
$$

Differentiating (3.4.1), we get

$$
\begin{aligned}
a_{i}^{\prime \prime}= & \text { (polynomial in the } a_{i} \text { 's and the }\left(a_{i}^{\prime}\right)^{\prime} \text { 's with } \\
& \text { coefficients in } C) / B\left(a_{1}, a_{2}, \ldots, a_{n}\right)^{2} .
\end{aligned}
$$

Using again (3.4.1), we obtain

$$
\left.a_{i}^{\prime \prime}=\text { (polynomial in the } a_{i} \text { 's with coefficients in } C\right) / B\left(a_{1}, a_{2}, \ldots, a_{n}\right)^{N} \text {. }
$$

Therefore by induction, 


$$
a_{i}^{(k)}=C\left[a_{1}, a_{2}, \ldots, a_{n}\right]_{B}
$$

for $1 \leq i \leq n$. Evaluating (3.4.3) at $x=Q$, we get

$$
a_{i}^{(k)}(Q) \in C\left(a_{1}(Q), a_{2}(Q), \ldots, a_{n}(Q)\right) .
$$

Namely every Taylor coefficient of the $a_{i}$ 's $(1 \leq i \leq n)$ with respect to the local parameter $x-Q$ is in $C\left(a_{1}(Q), a_{2}(Q), \ldots, a_{n}(Q)\right)$. Since the $a_{i}$ 's generate the field $K$ over $C$, for any $f \in K$ every Laurent coefficient of $f$ with respect to $x-Q$ is in $C\left(a_{1}(Q), a_{2}(Q), \ldots, a_{n}(Q)\right)$ (cf. Lemma (2.4)). Since each condition (1), (2), (3) is Zariski open, the lemma is proved.

If we understand $x-a$ to mean $1 / x$, it is not necessary to exclude the case where $p(Q)$ coincides with the point at infinity. But for our purpose the assertion of the lemma is sufficient.

Lemma (3.5). Let $p: R \rightarrow \mathbf{P}_{\mathbf{C}}^{1}$ be locally a covering morphism and $K$ a differential field of $\mathbf{Q}$-classical functions meromorphic on $R$. We assume that the field $K$ is finitely generated over $\mathbf{Q}(x)$. Then there exists a non-empty Zariski open set $W$ of $R$ such that for any $\mathbf{Q}^{\mathrm{c} 1}$-valued point $Q$ of $(W, \mathbf{Q}(x)),(1) p(Q)$ is not a point at infinity of $\mathbf{P}_{\mathbf{C}}^{1}$, (2) the morphism $p$ is not ramified at $Q$, (3) for any function $f \in \mathscr{O}_{Q}(K)$, the value $f(Q)$ is classical and such that $(4)$ the residue field $\mathscr{O}_{Q}(K) / m_{Q}(K)$ is finitely generated over $\mathbf{Q}$.

Proof. Since conditions (1), (2), (4) are Zariski open by Lemma (3.4), it is sufficient to find a non-empty Zariski open set $W$ satisfying (3). As the question is local, we may assume that $R$ is a non-empty open set of $\mathbf{P}_{\mathbf{C}}^{1}$. The argument of this case works for the general case. We prove the lemma by induction on the number of $G$-primitive operations to obtain $K$. If $K=\mathbf{Q}(x)$, we can take $W=R$. Now we use the notation of Definition (2.4) and assume there exists a non-empty Zariski open set $W$ of $S^{\prime}$ such that the lemma holds for $\left(S^{\prime}, \mathscr{K}\right)$. We may assume here $S^{\prime} \subset \mathbf{P}_{\mathbf{C}}^{1}$. For a moment let us further assume that the point $P$ of Definition (2.4.4) does not coincide with the point at infinity. The point $P$ is a $C_{\mathscr{K}}$-rational point of $\left(S^{\prime}, \mathscr{K}\right)$ and hence (the coordinate of) $P$ is a classical number. Multiplying a power of $x-P$ if necessary, we may assume that $f(x)$ is regular at $P$. In fact if $H(x)=$ $(x-P)^{m} f(x)$ is regular at $Q$ and if $H(Q)=(Q-P)^{m} f(Q)$ is a classical num. ber, then $f(Q)$ is a classical number since we may take $W$ so that $Q \neq P$ and since $P-Q$ is a classical number. Let $\mathcal{M}=\mathscr{K}(\{\varphi \circ F(v) \mid \varphi \in C(G)\})$. Let $U$ be an affine open set of $G$ such that $U_{L}$ is a neighbourhood of the point $v$. We show 
that for any $\varphi \in C[U]$, and for a $\mathbf{Q}$-valued point $Q \in\left(S^{\prime}, \mathbf{Q}(x)\right)$ which does not coincide with the point at infinity, $(\varphi \circ F(v))(Q)$ is $\mathbf{Q}$-classical. In fact we have

$$
f(Q)=\frac{1}{2 \pi i} \int_{\mathscr{C}} \frac{f(x)}{x-Q} d x
$$

for small circle $\mathscr{C}$ centered at $Q$. If we put $\mathcal{N}=\mathcal{M}(\pi i, Q)$, then $\mathcal{N}$ is a differential field of $\mathbf{Q}$-classical functions finitely generated over $\mathbf{Q}(x)$ and the point $P$ is a $C_{\mathcal{N}}$-valued point of $\left(S^{\prime}, \mathcal{N}\right)$ by Lemma $(2.7)$, where $C_{\mathcal{N}}$ denotes the field of constants of $\mathcal{N}$. Now we consider a differential equation for $g(x)$

$$
g^{\prime}(x)=\frac{f(x)}{2 \pi i(x-Q)} .
$$

with initial condition $g(P)=0$. Then the solution $g(x)$ is $\mathbf{Q}$-classical. In fact we take as an algebraic group $G$ the additive group $\mathbf{G}_{a}$ which is defined over $\mathbf{Q}$ hence over $C_{\mathcal{N}}$. We consider $\mathcal{N}(g)$-valued point $v: \operatorname{Spec} \mathcal{N}(g) \rightarrow \mathbf{G}_{a \mathcal{N}(g)}=$ $\operatorname{Spec} \mathcal{N}(g)[t]$ defined by $t \rightarrow g$. Then the meromorphic map $f(v): S^{\prime} \rightarrow \mathbf{G}_{a}^{\text {an }}=\mathbf{C}$ associated with the $\mathcal{N}(g)$-valued point $v$ is given by $x \rightarrow g(x)$. The point $Q$ is a $C_{\mathcal{N}}$-rational point of $\left(S^{\prime}, \mathcal{N}\right), F(v)(P)=g(P)=0 \in \mathbf{G}_{a C_{\mathcal{N}}}$ and $l \delta(v)=$ $g^{\prime}(x) \frac{d}{d x} \in$ Lie $G \otimes \mathcal{N}$. Precisely speaking we must consider a covering $q: \bar{S} \rightarrow$ $S^{\prime}$ so that the function $g(x)$ is single valued and the initial condition $g(P)$ should be read $g\left(P_{1}\right)=0$ for a point $P_{1} \in \bar{S}$ lying over $P$ (see Proof of Theorem (3.1)). We can replace the path $\mathscr{C}$ by a path $\mathscr{C}^{\prime}$ starting from $P$ and ending at $P$ as in the proof of Theorem (3.1). Then $f(Q)=\int_{\mathscr{C}} g^{\prime}(x) d x=\int_{\mathscr{C}^{\prime}} g^{\prime}(x) d x=g\left(P_{2}\right)-$ $g\left(P_{1}\right)=g\left(P_{2}\right)$ with a suitable point $P_{2} \in \bar{S}$ lying over $P$. We consider another solution $\bar{g}(x)$ of (3.5.1) with $\bar{g}\left(Q_{2}\right)=0$. Then $\bar{g}(x)$ is $\mathbf{Q}$-classical too. Therefore the difference $c=\bar{g}(x)-g(x)$ is $\mathbf{Q}$-classical and constant. The number $c=$ $\bar{g}\left(P_{2}\right)-g\left(P_{2}\right)=g\left(P_{2}\right)=f(Q)$ is classical. Now let us briefly treat the case where the point $P$ of Definition (2.4.4) coincides with the point at infinity. For any $\mathbf{Q}^{\text {cl }}$-valued point $Q$ of $\left(S^{\prime}, \mathbf{Q}(x)\right)$ with $Q \neq 0, \infty$, we show that $f(Q)$ is Q-classical. In fact $f(Q)=\frac{1}{2 \pi i} \int_{\mathscr{C}^{\prime}} \frac{f(1 / z)}{z-1 / Q} d z$ where $\mathscr{C}$ is a small circle centered at $z=1 / Q$. The argument is similar to the preceding case. We can replace the contour $\mathscr{C}$ by another contour $\mathscr{C}^{\prime}$ starting from $z=0$ and ending at $z=0$. We consider a differential equation

$$
\frac{d g}{d z}(z)=\frac{f(1 / z)}{2 \pi i(z-1 / Q)}
$$


which is equivalent to

$$
-x^{2}\left(\frac{d h}{d x}(x)\right)=\frac{f(x)}{2 \pi i(1 / x-1 / Q)}
$$

if we put $h(x)=g(z)$ and $x=1 / z$. Hence the differential equation (3.5.2) is equivalent to

$$
\frac{d h}{d x}(x)=\frac{f(x) Q}{2 \pi i(x-Q) x} .
$$

The integral $f(Q)=\frac{1}{2 \pi i} \int_{\mathscr{C}^{\prime}} \frac{f(1 / z)}{z-1 / Q} d z$ is equal to the difference $\bar{g}(z)$ $g(z)$ of integrals $\bar{g}(z), g(z)$ of (3.5.2) and hence to that of the integrals $\bar{h}(x)$, $h(x)$ of (3.5.3) satisfying rationality conditions at $\infty$. Since $\frac{f(x) Q}{2 \pi i(x-Q) x}$ is Q-classical and regular at $\infty$ which is a $C_{\mathcal{N}}$-valued point of $\left(S^{\prime}, \mathcal{N}\right)$, the argument of the preceding case proves that $f(Q)$ is classical.

We have $M=K M$ and the field $\mathcal{M}$ is the quotient field of $\mathscr{K}[\{\varphi \circ F(v) \mid \varphi \in$ $C(G)\}]$. Therefore there exist $f_{1}, f_{2}, \ldots, f_{m} \in K$ and $\varphi_{1}, \varphi_{2}, \ldots, \varphi_{n} \in C(G)$ such that $f_{1}, f_{2}, \ldots, f_{m}$ and $h_{1}=\varphi_{1}{ }^{\circ} F(v), h_{2}=\varphi_{2}{ }^{\circ} F(v), \ldots, h_{n}=\varphi_{n}{ }^{\circ} F(v)$ generate the field $M$ over the field $C_{M}$ of constants of $M$. We know that there exist a non-empty Zariski open set $V$ of $S^{\prime}$ such that $f_{1}, f_{2}, \ldots, f_{m}, h_{1}, h_{2}, \ldots, h_{n}$ are regular at $Q$ and $f_{1}(Q), f_{2}(Q), \ldots, f_{m}(Q), h_{1}(Q), h_{2}(Q), \ldots, h_{n}(Q)$ are classical for any $\mathbf{Q}^{\mathrm{cl}}$-valued point $Q \in(V, \mathbf{Q}(x))$. Now the lemma follows from Lemma (3.4).

Corollary (3.6). Let $p: R \rightarrow \mathbf{P}_{\mathbf{C}}^{1}$ be a locally a covering morphism of Riemann surfaces. Let $K$ be a differential field of $\mathbf{Q}$-classical functions meromorphic over $R$ such that the field $K$ is finitely generated over $\mathbf{Q}(x)$ (as an abstract field). Let $a_{1}, a_{2}, \ldots$, $a_{n} \in K$. Then there exists a non-empty Zariski open set of $R$ such that for any $\mathbf{Q}^{\mathrm{c} 1}$-valued point $Q$ of $(R, \mathbf{Q}(x))$, there exists a constant field $C^{\prime}$ consisting of classical numbers and finitely generated over $C_{K}$ (and hence over $\mathbf{Q}$ ) such that $(1) p(Q)$ is not the point at infinity of $\mathbf{P}_{\mathbf{C}}^{1}$, (2) the morphism $p$ is not ramified at $Q$, (3) $Q$ is a $C^{\prime}$-rational point of $\left(R, K C^{\prime}\right)$ and such that $(4) a_{1}, a_{2}, \ldots, a_{n}$ are regular at $Q$.

Proof. The intersection of the following three Zariski open sets satisfies our requirement if we take $C^{\prime}=\left\{f(Q) \mid f \in O_{Q}(K)\right\}$ :

(1) Zariski open set satisfying the condition (4) of the corollary,

(2) Zariski open set of Lemma (3.4),

(3) Zariski open set of Lemma (3.5). 
Theorem (3.7). Let $p: R \rightarrow \mathbf{P}_{\mathbf{C}}^{1}$ be locally a covering morphism of Riemann surfaces and let $f$ be a $\mathbf{Q}$-classical function meromorphic over $R$. Let $a \in R$ be $a$ $\mathbf{Q}^{\mathrm{cl}}$-valued point of $(R, \mathbf{Q}(x))$. If $f$ is regular at $a$, then $f(a)$ is a classical number.

Proof. Let us first prove the theorem under the additional assumption that the morphism $p: R \rightarrow \mathbf{P}^{1}$ is not ramified at the point $a$. We may assume that $R$ is a non-empty open set of $\mathbf{P}_{\mathbf{C}}^{1}$ and the morphism $p$ is the inclusion $R \subset \mathbf{C}$. Let us assume for a moment that the point $a$ does not coincide with the point at infinity of $\mathbf{P}_{\mathbf{C}}^{1}$. Then we have

$$
f(a)=\frac{1}{2 \pi i} \int_{\mathscr{C}} \frac{f(x)}{x-a} d x
$$

where $\mathscr{C}$ is a small circle centered at $a$. It follows from Corollary (3.6) that there exist a differential field $K$ finitely generated over $\mathbf{Q}(x)$ consisting of $\mathbf{Q}$-classical functions meromorphic over $R$ and $C_{K}$-rational point $Q \neq a$ of $(R, K)$ such that $\frac{f(x)}{2 \pi i(x-a)} \in K$ and such that $\frac{f(x)}{2 \pi i(x-a)}$ is regular at $Q$. We may replace the contour $\mathscr{C}$ by a path starting from $Q$ and ending at $Q$. Then the theorem for this case follows from the argument of Examples (2.13) and (2.14) and the proof of Lemma (3.5).

If $a$ is the point at infinity of $\mathbf{P}_{\mathbf{C}}^{1}$, then $f(a)=\frac{1}{2 \pi i} \int_{\mathscr{C}^{\prime}} \frac{f(1 / z)}{z-0} d z=\frac{1}{2 \pi i}$. $\int_{\mathscr{C}^{\prime}} \frac{f(x)}{x} d x$, where $\mathscr{C}^{\prime}$ is a small circle centered at $a=\infty$. Hence we can argue as above.

To treat that remaining case where the morphism $p: R \rightarrow \mathbf{P}_{\mathbf{C}}^{1}$ is ramified at the point $a$, we need

Sublemma (3.7.1). Let $p: R \rightarrow \mathbf{P}_{\mathbf{C}}^{1}$ be locally a covering morphism of Riemann surfaces and $f$ is a $\mathbf{Q}$-classical function meromorphic over $R$. Then a meromorphic function $\sqrt[n]{f}$ or any solution of an algebraic equation $y^{n}-f=0$ is $\mathbf{Q}$-classical.

Proof of Sublemma. The function $\sqrt[n]{f}$ is a solution of a linear differential equation $n f y^{\prime}-f^{\prime} y=0$. Then by Corollary (3.6), there exist a $\mathbf{Q}^{\mathrm{cl}}$-valued point $Q$ of $(R, \mathbf{Q}(x))$ and a field $K$ of $\mathbf{Q}$-classical functions meromorphic over $R$ such that (1) the field $K$ is finitely generated over $\mathbf{Q}(x)$, (2) $f \in K$, (3) $f$ is regular at $Q$, (4) $p(Q)$ is not the point at infinity of $\mathbf{P}_{\mathbf{C}}^{1}$, (5) the morphism $p$ is not ramified at $Q$, (6) $Q$ is a $C_{K^{-}}$valued point of $(R, K)$ and such that $f(Q) \neq 0$. If we consider a 
solution $g$ of $n f y^{\prime}-f^{\prime} y=0$ such that $g(Q)=\sqrt[n]{f(Q)}$, which is a classical num. ber by Corollary (3.2) is in $C_{K}$, then $g(x)$ is $\mathbf{Q}$-classical. This proves the sublemma (cf. Examples (1.7.2) and (2.13)).

Let us come back to the proof of the theorem when the morphism $p$ is ramified at the $\mathbf{Q}^{\mathrm{cl}}$-rational point $a$. Let us put $b=p(a)$. We may assume that $b$ is not the point at infinity of $\mathbf{P}_{\mathbf{C}}^{1}$ since the case where the point $b$ coincides with the point at infinity is treated similarly. Then $p^{*}(x-b)$ vanishes with order $n \geq 2$ at the point $a$. We denote simply $p^{*}(x-b)$ by $x-b$. Since $x-b$ is regular and vanishes with order $n$, replacing $R$ by an open neighbourhood of the point $a$, we may assume that there exists a holomorphic function $z$ on $R$ such that $z^{\mathrm{n}}=x-b$. Then $z$ is $\mathbf{Q}$-classical by Sublemma (3.7.1) and is a local parameter at the point $a$. Hence the function $f$ on $R$ is a function $g(z)$ of $z$ around the point $a$. Therefore $f(a)=\frac{1}{2 \pi i} \int_{\mathscr{C}} \frac{g(z)}{z-0} d z$, where $\mathscr{C}$ is a small circle centered at $a$. On the other hand

$$
f(a)=\frac{1}{2 \pi i} \int_{\mathscr{C}} \frac{g(z)}{z-0} d z=\frac{1}{2 \pi i} \int_{\mathscr{C}^{\prime}} \frac{g\left((x-b)^{1 / n}\right)}{(x-b)^{1 / n}} n x^{n-1} d x
$$

where $\mathscr{C}^{\prime}$ is the image of $\mathscr{C}$ under $p$. Now $g\left((x-b)^{1 / n}\right)$ as a function of $x$ is nothing but $f(x)$ and is $\mathbf{Q}$-classical. Therefore

$$
\left(\frac{1}{2 \pi i}\right) \frac{n x^{n-1} f(x)}{(x-b)^{1 / n}}
$$

is $\mathbf{Q}$-classical. The argument of unramified case shows that the value

$$
f(a)=\int_{\mathscr{C}^{\prime}}\left(\frac{1}{2 \pi i}\right) \frac{n x^{n-1} f(x)}{(x-b)^{1 / n}} d x
$$

is classical.

THEOREM (3.8). Let $p: R \rightarrow \mathbf{P}_{\mathbf{C}}^{1}$ be locally a covering morphism of Riemann sur. faces. Let $f(x)$ be a $\mathbf{Q}$-classical function meromorphic on $R$ such that a 1-form $p^{*} f(x) d x$ is regular on $R$. Let $a, b \in R$ be $\mathbf{Q}^{\text {cl }}$-valued points of $(R, \mathbf{Q}(x))$ of $\mathbf{P}^{1}$. Then the definite ntegral $\int_{a}^{b} f(x) d x$ is a classical number for any path joining $a$ and $b$.

Proof. It follows from Corollary (3.6) that there exist a differential field $K \supset$ $\mathbf{Q}(x)$ of $\mathbf{Q}$-classical functions meromorphic over $R$ and a $C_{K^{-}}$valued point $P$ of $(R, K)$ such that the function $f$ belongs to $K$, morphism $p$ is not ramified at $P$, $p(P)$ is not the point at infinity of $\mathbf{P}^{1}, p^{*} f d x$ is regular at $P$ and such that the 
field $K$ is finitely generated over $\mathbf{Q}(x)$. Since $\int_{a}^{b} f(x) d x=\int_{a}^{P} f(x) d x-$ $\int_{b}^{P} f(x) d x$, we may assume $b=P$. Let $q: S \rightarrow R$ be the universal covering space of $R$. Let $P_{1} \in S$ be a point lying over $P$. Consider a differential equation $g^{\prime}(x)=$ $f(x)$ with initial condition $g\left(P_{1}\right)=0$. Then $g(x)$ is $\mathbf{Q}$-classical (cf. Example (2.14)). There exists a point $Q \in S$ such that $\int_{a}^{b} f(x) d x=g\left(P_{1}\right)-g(Q)=$ $g(Q)$ which is classical by Theorem (3.7).

Here are applications of Theorem (3.8).

Examples (3.9.1). The period $K$ which appears in the theory of elliptic functions is classical if $\kappa$ is classical. Namely $K$ is defined by the following formula

$$
K=\frac{1}{2} \int_{0}^{1} \frac{d x}{\left[x(1-x)\left(1-\kappa^{2} x\right)\right]^{1 / 2}} \quad(\kappa \neq 0, \pm 1) .
$$

The function $\left[x(1-x)\left(1-\kappa^{2} x\right)\right]^{1 / 2}$ of $x$ is $\mathbf{Q}$-classical by Sublemma (3.7.1) if $\kappa$ is a classical number. If we consider an elliptic curve $R: y^{2}=x(1-x)(1-$ $\left.k^{2} x\right)$, then $\frac{d x}{\left[x(1-x)\left(1-\kappa^{2} x\right)\right]^{1 / 2}}$ is regular on $R$ and we can apply Theorem (3.8).

(3.9.2) The Eulerian integral $B(m, n)=\int_{0}^{1} x^{m-1}(1-x)^{n-1} d x$ which is equal to $\frac{\Gamma(m) \Gamma(n)}{\Gamma(m+n)}$ is $\mathbf{Q}$-classical for $m, n \in \mathbf{Q}, m, n>0$, where $\Gamma(x)$ denotes the gamma function. Here $x^{m-1},(1-x)^{n-1}$ are understood to mean values of $e^{(m-1) \log x}$ and $e^{(n-1) \log (1-x)}$ (cf. [WW] Chap. XII, 12.4). In fact first let us assume $m$, $n \geq 1$ and put $r / t=m-1, s / t=n-1$ with $r, s, t \in \mathbf{N}, t \geq 1$ and $(r, s$, $t)=1$. The polynomial $y^{t}=x^{r}(1-x)^{s}$ is irreducible in $\mathbf{C}[x, y]$. Let $p: R \rightarrow \mathbf{P}_{\mathbf{C}}^{1}$ be the Riemann surface of $y^{t}=x^{r}(1-x)^{s}$. Then $p^{*} x^{m-1}(1-x)^{n-1} d x$ is regular on $R=p^{-1}(\mathbf{C})$. It follows from Theorem (3.8) that $B(m, n)$ is classical for $m, n$ $\in \mathbf{Q}$ if $m, n \geq 1$. Since we have $B(p, q)=B(q, p)$ and $B(p, q+1)=\frac{q}{p+q}$ $B(p, q)$ for $p, q \in \mathbf{C}$ with $R(p), R(q)>0$ where $R(z)$ denotes the real part of a number $z$ (cf. [WW] Chap. XII, 12.4). Hence $B(m, n)$ is classical if $m, n \in \mathbf{Q}$ and $m, n>0$ (see $\S 6,(6.13)$ where the values of the gamma function are discussed). 
In view of Example (3.9.2) it is natural to ask the following question.

Problem (3.9.3). If $m, n$ are positive classical numbers, $B(m, n)$ is classical?

We show that the field of $\mathbf{Q}$-classical functions is algebraically closed. To this end we need

Proposition (3.10). Let $G$ be an algebraic group defined over a field $C$ of classical numbers. Let $D \subset \mathbf{C}$ be a domain and $F: D \rightarrow G \otimes_{C} \mathbf{C}$ be an analytic map. For any $\varphi \in C(G), \varphi^{\circ} F$ is a $\mathbf{Q}$-classical function if the following conditions are satisfied.

(i) Let $v: \operatorname{Spec} \mathbf{C}(D) \rightarrow G_{\mathbf{C}}(D)$ be a $\mathbf{C}(D)$-valued point of $G_{\mathbf{C}(D)}$ corresponding to $F$ (cf. Remark (1.2)). Then $l \delta(v) \in$ Lie $G \otimes_{c} \mathbf{C}(D)$ lies in Lie $G \otimes_{c} K$ for suitable differential subfield $C \subset K \subset \mathbf{C}(D)$ of $\mathbf{Q}$-classical functions with $\mathbf{Q}(x) \subset K$ $(\mathbf{C}(D)$ denotes the differential field of the meromorphic functions on $D))$.

(ii) There exists a field extension $C^{\prime} \supset C$ of finite type in $\mathbf{Q}^{\mathrm{cl}}$ and $C^{\prime}$-valued point $P$ of $(D, K)$ such that $F$ is regular at $P$ and such that $F(P) \in G \otimes_{C} \mathbf{C}$ arises from a $\mathbf{Q}^{\mathrm{cl}}$-rational point of $G \otimes_{C} \mathbf{Q}^{\mathrm{c} 1}$.

Proof. In fact we may assume that the field $K$ is finitely generated over $\mathbf{Q}(x)$ so that also the field $C$ is finitely generated over $\mathbf{Q}$. If these conditions are satisfied, then by Lemma (2.3) we can find a differential field extension $\mathscr{K} \supset K$ in $\mathbf{C}(D)$ obtained by an adjonction of a finite number of classical numbers to $K$ such that $G$ is defined over the field $C_{\mathscr{K}}$ of the constants of $\mathscr{K}, l \delta(v) \in \operatorname{Lie} G \otimes_{C_{\mathscr{K}}} \mathscr{K}, P$ is a $C_{\mathscr{K}}$-valued point of $(D, \mathscr{K})$ and such that $F$ is regular at $P$ and $F(P) \in G$ $\otimes_{C_{\mathscr{K}}} \mathbf{C}$ arises from a $C_{\mathscr{K}}$-valued point of $G$. The field $\mathscr{K}$ is finitely generated over $\mathbf{Q}(x)$ and consists of $\mathbf{Q}$-classical functions meromorphic over $D$. Hence by step (4) of Definition (2.4), $\varphi \circ F$ is $\mathbf{Q}$-classical.

Corollary (3.11). Let $D \subset \mathbf{C}$ be a domain and $M$ be a differential field of Q-classical functions meromorphic over $D$. We assume that the field $M$ is finitely generated over $\mathbf{Q}(x)$. Let $a_{1}, a_{2}, \ldots, a_{n} \in M$ and let us consider a linear differential equation

$$
\frac{d^{n} y}{d x^{n}}+a_{1} \frac{d^{n-1} y}{d x^{n-1}}+\cdots+a_{n} y=0 .
$$

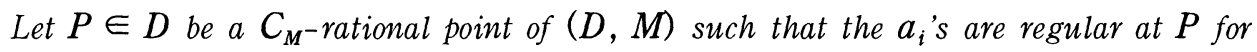
$1 \leq i \leq n$ and let $f$ be a solution of $(*)$. If $f(P), f^{\prime}(P), \ldots, f^{(n-1)}(P)$ are classical numbers, then $f, f^{\prime}, \ldots, f^{(n-1)}$ are $\mathbf{Q}$-classical functions. 
Proof. Let us put

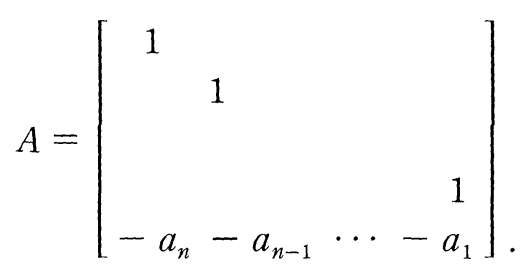

Then a function $g(x)$ is a solution of $(*)$ if we have

$$
\left[\begin{array}{l}
g^{\prime} \\
g^{\prime \prime} \\
\vdots \\
g^{(n)}
\end{array}\right]=A\left[\begin{array}{l}
g \\
g^{\prime} \\
\vdots \\
g^{(n-1)}
\end{array}\right]
$$

We can find $C$-linearly independent solutions $f(x), f_{1}(x), \ldots, f_{n-1}(x)$ of $(*)$ such that the Wronskian

$$
\left|\begin{array}{cccc}
f & f_{1} & \cdots & f_{n-1} \\
f^{\prime} & f_{1}^{\prime} & \cdots & f_{n-1}^{\prime} \\
\vdots & \vdots & & \vdots \\
f^{(n-1)} & f_{1}^{(n-1)} & \cdots & f_{n-1}^{(n-1)}
\end{array}\right| \neq 0
$$

and such that

$$
f_{j}^{(k)}(P) \text { is Q-classical for } 1 \leq j \leq n-1,0 \leq k \leq n-1 .
$$

We thus get a map $F: D \rightarrow \mathbf{G L}_{n}$,

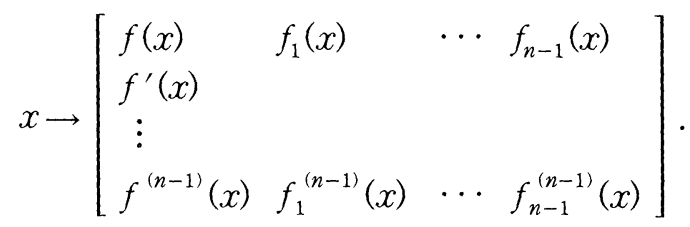

Let $v: \operatorname{Spec} \mathbf{C}(D) \rightarrow \mathbf{G L}_{n} \mathbf{C}(D)$ be the corresponding $\mathbf{C}(D)$-rational point. Then we know $l \delta(v)=F^{\prime} F^{-1}=A$. It follows from Proposition (3.10), for $K=M$ and $C^{\prime}$ $=C_{M}\left(f_{j}^{(k)}(P)\right)_{0 \leq j, k \leq n-1}$ if we take as $\varphi \in C\left(\mathbf{G L}_{n c}\right)$ a regular function $\varphi_{i j}\left(\left(a_{i j}\right)\right)=a_{i j}$ of taking the value of $(i j)$-component of the matrix $\left(a_{i j}\right)$. Here we understand $f_{0}^{(k)}=f^{(k)}$ for $0 \leq k \leq n-1$. Then $\varphi^{\circ} F$ is $\mathbf{Q}$-classical. In particular $\varphi_{j 0}^{\circ} F=f^{(j)}$ is $\mathbf{Q}$-classical for $j \in \mathbf{N}$.

Proposition (3.12). Let $a_{1}, a_{2}, \ldots, a_{n}$ be $\mathbf{Q}$-classical functions. Then a solution $f$ of an algebraic equation $f^{n}+a_{1} f^{n-1}+\cdots+a_{n}=0$ is $\mathbf{Q}$-classical. 
Proof. We may assume that the polynomial $H(X)=X^{n}+a_{1} X^{n-1}+\cdots+$ $a_{n} \in \mathbf{Q}(x)\left\langle a_{1}, a_{2}, \ldots, a_{n}\right\rangle[X]$ is irreducible $\left(\mathbf{Q}(x)\left\langle a_{1}, a_{2}, \ldots, a_{b}\right\rangle=\mathbf{Q}\left(x, a_{1}\right.\right.$, $\left.a_{2}, \ldots, a_{n}, a_{1}^{\prime}, a_{2}^{\prime}, \ldots, a_{n}^{\prime}, a_{1}^{\prime \prime}, a_{2}^{\prime \prime}, \ldots\right)$ by definition). As $H(X)$ is the minimal polynomial of $f$ over $\mathbf{Q}(x)\left\langle a_{1}, a_{2}, \ldots, a_{n}\right\rangle, H^{\prime}(f)=f^{n-1}+(n-1) a_{1} f^{(n-2)}+\cdots$ $+a_{n-1} \neq 0$. Then $\left(\mathbf{Q}(x)\left\langle a_{1}, a_{2}, \ldots, a_{n}\right\rangle(f): \mathbf{Q}(x)\left\langle a_{1}, a_{2}, \ldots, a_{n}\right\rangle\right)=n$. Since $f$ is algebraic over $\mathbf{Q}(x)\left\langle a_{1}, a_{2}, \ldots, a_{n}\right\rangle, \mathbf{Q}(x)\left\langle a_{1}, a_{2}, \ldots, a_{n}\right\rangle(f)$ is a differential field. Therefore $f, f^{\prime}, \ldots, f^{(n)}$ are linearly dependent over $\mathbf{Q}(x)\left\langle a_{1}, a_{2}, \ldots, a_{n}\right\rangle$ : there exist $b_{0}, b_{1}, \ldots, b_{n} \in \mathbf{Q}(x)\left\langle a_{1}, a_{2}, \ldots, a_{n}\right\rangle$ such that $b_{0} f^{(n)}+b_{1} f^{(n-1)}+\cdots$ $+b_{n} f=0$ with $\left(b_{0}, b_{1}, \ldots, b_{n}\right) \neq 0$. Let

$$
f^{(m)}+e_{1} f^{(m-1)}+\cdots+e_{m} f=0
$$

be a linear differential equation satisfied by $f$ with $e_{i} \in \mathbf{Q}(x)\left\langle a_{1}, a_{2}, \ldots, a_{n}\right\rangle$ for $1 \leq i \leq m$. It follows from Lemma (3.5) and Corollary (3.6) that there exist a domain $D$ of $\mathbf{C}$, a differential field $K \supset \mathbf{Q}(x)$ of $\mathbf{Q}$-classical functions meromorphic on $D$ and a $C_{K}$-valued point $P \in(D, K)$ such that (1) $K$ is finitely generated over $\mathbf{Q}(x),(2)$ the $a_{i}$ 's $(1 \leq i \leq n)$ belong to $K$, (3) the $a_{i}$ 's and the $\boldsymbol{e}_{j}$ 's are regular at $P$ for $1 \leq i \leq n, 1 \leq j \leq m$, (4) $\left(n f^{n-1}+(n-1) a_{1} f^{n-2}+\cdots+a_{n-1}\right)(P) \neq 0$ and such that $(5)$ the subfield $\mathscr{O}_{P}(K) / m_{P}(K) \subset \mathbf{C}$ consists of $\mathbf{Q}$-classical numbers. The value $f(P)$ is algebraic over $\mathbf{Q}(P)\left(a_{1}(P), a_{2}(P), \ldots, a_{n}(P)\right)$ and hence classical by Corollary (3.2) and Theorem (3.7). Since $\left(n f^{n-1}+(n-1) a_{1} f^{n-1}+\right.$ $\left.\cdots+a_{n-1}\right) f^{\prime}+f^{n}+a_{1}^{\prime} f^{n-1}+\cdots+a_{n}^{\prime}=0$,

$$
f^{\prime}=\frac{f^{n}+a_{1}^{\prime} f^{n-1}+\cdots+a_{n}^{\prime}}{n f^{n-1}+(n-1) a_{1} f^{n-2}+\cdots+a_{n-1}},
$$

and hence

$$
\begin{aligned}
& f^{\prime}(P) \in \mathbf{Q}\left[f(P), a_{1}(P), a_{2}(P), \ldots, a_{n}(P), a_{1}^{\prime}(P), a_{2}^{\prime}(P), \ldots,\right. \\
&\left.a_{n}^{\prime}(P), \frac{1}{n f^{n-1}+(n-1) a_{1} f^{n-2}+\cdots+a_{n-1}}(P)\right] \\
& \subset \mathcal{O}_{P}(K) / m_{P}(K)[f(P)](\subset \mathbf{C}) .
\end{aligned}
$$

Hence $f^{\prime}(P)$ is classical. For the similar reason $f^{\prime \prime}(P), f^{(3)}(P), \ldots \in \mathscr{O}_{P}(K) /$ $m_{P}(K)[f(P)]$ and are classical. Therefore by Corollary (3.11), $f(x)$ is $\mathbf{Q}$-classical.

We recall the following lemma which we use in the proof of Theorem (3.14).

Lemma (3.13). Let $L$ be a differential field, $C$ a field of constants of $L$ and $G$ an algebraic group defined over $C$. Let $u$ and $v$ be L-rational points of $G_{L}=G \otimes_{C} L$. 
Then we have

$$
l \delta(u v)=l \delta(u)+L_{u *} l \delta(v),
$$

where $u v \in G_{L}$ is the product of the L-valued points $u$ and $v$ by the group law of $G_{L}$ and $L_{u}$ is the left translation $G_{L} \rightarrow G_{L}$ by the $L$-rational point $u$.

Lemma (3.13) is proved in [U2] (Lemma 16).

Remark. The equality $(*)$ shows that the map $l \delta: G_{L}(L) \rightarrow$ Lie $G_{L}, u \rightarrow$ $l \delta(u)$ satisfies the 1 -cocycle condition if we regard the vector space $L_{i e} G_{L}$ as a $G_{L}(L)$-module by the left translation (= the adjoint representation).

THEOREm (3.14). In Definition (2.4) of $\mathbf{Q}$-classical functions, we may assume that $G$ is either the general linear group $\mathbf{G L}_{n}(n \geq 1)$ or an abelian variety.

Proof. The proof is based on the same idea as in [U4] where we introduced the classical functions and proved a similar assertion for the classical functions. Since a linear algebraic group defined over a field $k$ is a closed subgroup of the general linear group $G L_{n k}$, it is sufficient to prove that we may assume $G$ is either a linear algebraic group or an abelian variety. We start from $\mathbf{Q}(x)$. Let $K$ be a field of $\mathbf{Q}$-classical functions which are already defined by induction. We assume that the theorem holds for $K$ or $K$ is contained in successive $G$-primitave extensions of Definition (2.4) where $G$ is either a linear algebraic group or an abelian variety. There exists locally a covering morphism $p: R \rightarrow \mathbf{P}_{\mathbf{C}}^{1}$ of Riemann surfaces such that field $K$ is a field of meromorphic functions on $R$. Let $C$ be the field of the constants of $K, \mathscr{K}$ a differential subfield of $K$ with $\mathscr{K} \supset \mathbf{Q}(x)$ and $\mathscr{K} \supset C$ and $G$ an algebraic group defined over $C$. Let $q: S \rightarrow R$ be locally a covering morphism of Riemann surfaces. Let $L$ be a differential field of meromorphic functions over $S$ with derivation $d / d x$ such that the field $K$ is identified with a differential subfield of $L$ by $q^{*}$. Let $v: \operatorname{Spec} L \rightarrow G_{L}$ be an $L$-rational point of $G_{L}$ satisfying the following conditions (a) and (b).

(a) $l \delta(v)$ which is an element of Lie $G \otimes_{C} L$ lies in Lie $G \otimes_{C} \mathscr{K}$.

(b) There exist a Riemann surface $S^{\prime}$ and a point $P$ of $S^{\prime}$ such that (1) $S^{\prime}$ contains $S$ as an open subspace, (2) any element of $\mathscr{K}=q^{*} \mathscr{K}$ is meromorphic on $S^{\prime}$, (3) the point $P$ is a $C$-valued point of $\left(S^{\prime}, \mathscr{K}\right),(4)$ the meromorphic map $F(v): S^{\prime}$ $\cdots \rightarrow G \otimes_{C} \mathbf{C}$ is regular at $P$ and such that (5) the image $F(v)(P)$ is a $C$-rational point of $G$. Then $\left.L^{\prime}=K\left(\varphi \circ F(v) \mid \varphi \in K\left(G_{K}\right)\right\}\right)=K(\{\varphi \circ F(v) \mid \varphi \in$ $\left.C\left(G_{C}\right)\right\}$ ), which is a differential subfield of $L$, is a newly defined $Q$-classical func- 
tions. We show that the field $L^{\prime}$ is obtained by a composite of $H$-primitive extensions with rationality conditions where the algebraic group $H$ is either a linear algebraic group or an abelian variety. We may assume that $G$ is neither a linear algebraic group nor an abelian variety. By a theorem of Chevalley (see [R1], Theorem 16), there exists a closed linear normal subgroup $N$ defined over $C$ of $G$ such that the quotient $A=G / N$ is an abelian variety:

$$
1 \rightarrow N \rightarrow G \stackrel{p}{\rightarrow} A \rightarrow 0 \text {. }
$$

The composite $\mu_{L} \circ v: \operatorname{Spec} L \rightarrow G_{L} \rightarrow A_{L}$ is an $L$-rational point of $A_{L}$. Since $\mu_{*} l \delta(v)=l \delta\left(\mu_{L}{ }^{\circ} v\right)$ by Lemma 15 in [U2] and since $l \delta(v) \in$ Lie $G \otimes_{C} \mathscr{K}, l \delta\left(\mu_{L}^{\circ} v\right)$ lies in Lie $A \otimes_{C} \mathscr{K} . \mu_{C}{ }^{\circ} F(v)(P) \in A_{C}$ comes from a $C$-rational point of $A$ since the point $F(v)(P)$ and the morphism $p$ are defined over $C$. Therefore $M=K(\{\varphi \circ$ $\left.\left./{ }_{\mathbf{C}} \circ F(v) \mid \varphi \in K\left(A_{K}\right)\right\}\right)=K\left(\left\{\varphi \circ / \mathbf{C}^{\circ} F(v) \mid \varphi \in C\left(A_{K}\right)\right\}\right)$ is a differential field of Q-classical functions constructed by the abelian variety $A$. Let us denote $F(v)$ simply by $F$ and put $\left.\left.\mathcal{M}=\mathscr{K}\left(\varphi \circ /{ }_{c}{ }^{\circ} F \mid \varphi \in \mathscr{K}\left(A_{\mathscr{K}}\right)\right\}\right)=\mathscr{K}\left(\varphi^{\circ} /{ }_{c}{ }^{\circ} F \mid \varphi \in C\left(A_{C}\right)\right\}\right)$. Since $\mathcal{M}$ is an $A$-primitive extension of $\mathscr{K}, \mathcal{M}$ is a differential subfield of $L$ (cf. [U2], Remark 18.1). The point $P \in S^{\prime}$ is a $C$-rational point of $\left(S^{\prime}, \mathcal{M}\right)$ by Lemma (2.6). Let us assume for a moment that $p$ has a $C$-rational section $s: A \cdots \rightarrow G: \not \circ s=\operatorname{Id}_{A}$. We show that an extension $L^{\prime} \supset M$ is defined by linear algebraic groups. We may assume by Corollary (3.3), whose proof involves only the additive group $\mathbf{G}_{\boldsymbol{a}}$ that

(3.14.1) we can replace the field $C$ of the constants of $K$ by a finite algebraic extension.

By (3.14.1) and by considering a translation by a $C$-rational point, we may assume that $s$ is regular at $\mu \circ v(\operatorname{Spec} L)$. Let $F_{1}: S \cdots \rightarrow G_{\mathbf{C}}^{\text {an }}$ be a meromorphic map associated with $v_{1}=s \circ p \circ v: \operatorname{Spec} L \rightarrow G$ so that we have $F_{1}=s \circ p \circ F$. Then $v v_{1}^{-1}$ is a $L$-rational point of $N_{L}$ and $\not \circ F_{1}=\not \circ F$. We may assume by a translation by a $C$-rational point that $F_{1}$ is regular at $P$. For any $\varphi \in C\left(G_{C}\right), \varphi \circ F_{1}=\varphi \circ(s \circ \mu \circ F)$ $=(\varphi \circ s) \circ(\not \circ F)$ is in $\mathcal{M}$ since $(\varphi \circ s) \in C\left(A_{C}\right) \cdot 0=l \delta\left(v_{1}^{-1} v_{1}\right)=l \delta\left(v_{1}^{-1}\right)+$ $L_{v_{1}^{-1} *}\left(l \delta\left(v_{1}\right)\right)$ by Lemma (3.13) and hence $l \delta\left(v_{1}^{-1}\right) \in$ Lie $G \otimes_{c} \mathcal{M}$ since $v_{1}$ is a $\mathcal{M}$-valued point. We have $l \delta\left(v_{1}^{-1} v\right)=l \delta\left(v_{1}^{-1}\right)+L_{v_{1}^{-1} *} l \delta(v)$ by Lemma (3.12) and hence $l \delta\left(v_{1}^{-1} v\right) \in$ Lie $G_{C} \otimes \mathcal{M}$. The point $F_{1}(P)$ and hence the point $F_{1}(P)^{-1} F(P)$ come from $\mathrm{C}$-valued points of $G_{C}$ and by Lemma (2.6), $P$ is a $C$-valued point of $\left(S^{\prime}\right.$, $M)$. Since $l \delta\left(v_{1}^{-1} v\right) \in$ Lie $G \otimes_{C} \mathcal{M} \cap$ Lie $N \otimes_{C} L=$ Lie $N \otimes_{C} \mathcal{M}$, the extension $L^{\prime}=$ $K(v)=K\left(v_{1}, v_{1}^{-1} v\right)=K\left(v_{1}\right)\left(v_{1}^{-1} v\right) \supset M=K\left(v_{1}\right)$ is an $N$-primitive extension satisfying the condition (4) of Definition (2.4) and the theorem is proved in this particular case.

Let us now consider the general situation where there is no $C$-rational section $s: A \cdots \rightarrow G$. In this case we can find a finite algebraic field extension $C^{\prime}$ of $C$, a fi- 
nite $C^{\prime}$-morphism $q: \tilde{A} \rightarrow A_{C^{\prime}}$ of algebraic varieties and a $C^{\prime}$-rational map $s^{\prime}$ : $\tilde{A} \cdots \rightarrow G_{C^{\prime}}$ such that $\mu_{C^{\prime}} \circ s^{\prime}=q$. We may assume $C=C^{\prime}$ by Corollary (3.2) since its proof involves only a linear algebraic group. We have an $L$-rational point $/ L_{L}^{\circ} v$ : Spec $L \rightarrow \mathbf{G}_{L} \rightarrow A_{L}$. For the same reason as in the preceding case, $M=K\left(\left\{\varphi^{\circ} p^{\circ} F \mid\right.\right.$ $\left.\left.\varphi \in K\left(A_{k}\right)\right\}\right) \supset K$ is a differential field of $\mathbf{Q}$-classical functions constructed from $K$ by the abelian variety $A$. Similarly if we put $\mathcal{M}=\mathscr{K}(\{\varphi \circ p \circ F \mid \varphi \in C(A)\}) \supset$ $\mathscr{K}, \mathcal{M}$ is a differential field of $\mathbf{Q}$-classical functions. Since $q: \tilde{A} \rightarrow A_{C^{\prime}}$ is finite, there exist a finite algebraic field extension $\tilde{M} \supset M$ and a morphism $w: \operatorname{Spec} \bar{M}$ $\rightarrow \tilde{A}$ such that the diagram

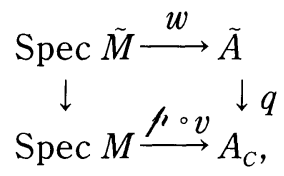

is commutative. Here the left vertical arrow is induced from the inclusion $\tilde{M} \supset M$. The restriction $v \mid \operatorname{Spec} M$ is denoted again by $v . \tilde{M}$ consists of $\mathbf{Q}$-classical functions obtained from $M$ by a linear algebraic group by Proposition (3.12). We may assume that since the extension $\tilde{M} \supset M$ is finite algebraic, $\tilde{M}$ is a subfield of $L, s^{\prime}$ is regular at $w(\operatorname{Spec} \tilde{M})$ and that $q: \tilde{A} \rightarrow A_{C^{\prime}}$ is unramified over $p \circ F(P)$ as in the preceding case. Hence we may assume that $\tilde{M}$ is a field of meromorphic functions over a small disc on $S^{\prime}$ centered at $P$. Let us define $\bar{v}_{1}$ by composite $\bar{v}_{1}$ : $\operatorname{Spec} L \rightarrow \operatorname{Spec} \tilde{M} \stackrel{w}{\rightarrow} \tilde{A} \stackrel{s}{\rightarrow} \rightarrow G$. The corresponding $L$-rational point $\operatorname{Spec} L \rightarrow$ $G_{L}$ is denoted by $v_{1}$. We may assume that $F\left(v_{1}\right): S^{\prime} \cdots \rightarrow G_{C}$ is regular at $P$. The point $\left.P \in S^{\prime}, \mathcal{M}\right)$ may not be $C$-rational. In that case by adding classical numbers to $\tilde{M}$ by Corollary (3.2) which involves only a linear algebraic group, we may assume that $P$ is a $C_{\tilde{M}}$-rational point (we notice that $\mathscr{O}_{P}(\tilde{M}) / m_{P}(\tilde{M})$ is a finite algebraic extension of $\mathscr{O}_{P}(M) / m_{P}(M)$ (cf. [Z] Ch. VI, $\S 6$, Corollary 2)). It is sufficient to show that the extension $L^{\prime}=\tilde{M}(v) \supset \tilde{M}$ is $N$-primitive satisfying the rationality conditions of Definition (2.4). Now we have $2 L$-rational points $v$ and $v_{1}$ of $G_{L} . l \delta(v) \in$ Lie $G \otimes \mathscr{K}$ by induction hypotheses and hence is an element of Lie $G \otimes \mu \subset$ Lie $G \otimes \tilde{M}$. Since $v_{1}$ comes from an $\tilde{M}$-rational point of $G_{\tilde{M}}$, $l \delta\left(v_{1}\right) \in$ Lie $G \otimes \tilde{M}$. Therefore by the argument of the preceding case, we can prove

$$
l \delta\left(v_{1} v^{-1}\right) \in \operatorname{Lie} G \otimes \tilde{M} .
$$

Since the point $P \in\left(S^{\prime}, \tilde{M}\right)$ is $C_{\tilde{M}}$-rational, the extension $L^{\prime}=\tilde{M}(v)=\bar{M}(v$, $\left.v_{1}^{-1}\right)=\tilde{M}\left(v_{1}^{-1} v\right) \supset \tilde{M}$ is an $N$-primitive extension satisfying the condition (4) of Definition (2.4) and the theorem is proved.

Let $D_{1}, D_{2}$ be domains of $\mathbf{C}$ and $f, g \mathbf{Q}$-classical functions meromorphic on $D$ 
and $E$. If $f$ is regular on $D_{1}, f\left(D_{1}\right) \subset D_{2}$ and if the image $f\left(D_{1}\right)$ is not contained in the pole of $g$, we can speak of the composite function $g \circ f$.

THeOREm (3.15). If $f$ and $g$ are $\mathbf{Q}$-classical, then the composite $g \circ f$ is $\mathbf{Q}$-classical.

Proof. We may assume by Theorem (3.7) that $f$ is not constant. We prove the theorem by induction on the number of steps to obtain $g$. If $g$ is in $\mathbf{Q}(x)$, the theorem holds. By Theorem (3.13) it is sufficient to check the theorem for a $G$-primitive extension under the assumption that $G$ is either the general linear group or an abelian variety.

(3.16) $G$ is the general linear group $\mathbf{G L}_{n}$. In this case the field extension is given by an adjonction of a $\mathbf{Q}$-classical solution $F(x)=\left(f_{i j}(x)\right)_{1 \leq i, j \leq n}$ of a linear differential equation

$$
F^{\prime}(x)=A(x) F(x)
$$

with $A(x)=\left(a_{i j}(x)\right) \in M_{n}(K)$ where $K$ is a field of known $Q$-classical functions meromorphic over $D_{2}$ and $K$ is finitely generated over $\mathbf{Q}(x)$. We assume that the $\left(h \circ f\right.$ )'s are $\mathbf{Q}$-classical for $h \in K$ and we have to prove that the $f_{i j}(f)$ 's are Q-classical. Since $f_{i j}(f)^{\prime}=f_{i j}^{\prime}(f) f^{\prime}$, we have

$$
F(f)^{\prime}=f^{\prime} A(f) F(f) .
$$

We put $L=\mathbf{Q}(x)\left\langle f, a_{i j}(f), f_{i j}(f)\right\rangle_{1 \leq i, j \leq n}, K=\mathbf{Q}(x)\left\langle f, a_{i j}(f)\right\rangle_{1 \leq i, j \leq n}$. Namely $L$ (resp. $K$ ) is the differential field generated by $f$, the $a_{i j}(f)$ 's and the $f_{i j}(f)$ 's (resp. by $f$ and the $a_{i j}(f)$ 's) over $\mathbf{Q}(x)$. By Lemma (3.5) there exist a $\mathbf{Q}^{\text {cl }}$-valued point $P$ of $\left(D_{1}, \mathbf{Q}(x)\right)$ such that $f$ and the $a_{i j}(f)$ 's are regular at $P, F(f(P)) \in$ $\mathbf{G L}_{n}(\mathbf{C})$ and such that if we put $C^{\prime}=\left\{h(p) \mid h \in \mathscr{O}_{P}(K)\right\}$, then the field $C^{\prime}$ consists of classical numbers and is finitely generated over $\mathbf{Q}$. We put $\mathscr{K}=C^{\prime}(x)\langle f$, $\left.a_{i j}(f)\right\rangle_{1 \leq i, j \leq n}$. Then $P$ is a $C^{\prime}$-valued point of $\left(D_{1}, \mathscr{K}\right)$. Since the $f_{i j}(f(P))$ 's are classical numbers by Theorem (3.7) and $F(f)$ satisfies the differential equation (*), therefore the $f_{i j}(f)$ 's are $\mathbf{Q}$-classical by Corollary (3.11).

(3.17) Now we treat an $A$-primitive extension $L / K$ for an abelian variety $A$. Namely $K$ is a differential field of known $\mathbf{Q}$-classical functions meromorphic on $D_{2}$ and the field $K$ is finitely generated over $\mathbf{Q}(x)$. We assume that $h \circ f$ is Q-classical for $h \in K$. The abelian variety $A$ is defined over the field $C_{K}$ of constants of $K$. Let $X_{1}, X_{2}, \ldots, X_{d}$ be $C_{K^{-}}$-linearly independent vector fields on $A$ with $d=\operatorname{dim} A$. There exists a universal covering space $\pi: \mathbf{C}^{d} \rightarrow A_{\mathbf{C}}$ such that $\pi_{*}(\partial /$ $\left.\partial x_{i}\right)=X_{i}$ for $1 \leq i \leq d$. Let $v: \operatorname{Spec} L \rightarrow A_{L}$ be an $L$-rational point defining the $A$-primitive extension $L / K$. Let $F=F(v): D_{2} \rightarrow A_{\mathbf{C}}^{\text {an }}$ be the associated meromor- 
phic map. We may assume that $F$ is regular, the map $F$ factors through $\pi$ and that there exists a regular map $\mathscr{F}: D_{2} \rightarrow \mathbf{C}^{d}$ such that $\pi \circ \mathscr{F}=F$. It follows from our assumption that (1) $l \delta(v) \in$ Lie $A_{L}=$ Lie $A \otimes_{C} L$ lies in Lie $A \otimes_{C} K$ and that (2) $L=K(\{\varphi \circ F \mid \varphi \in C(A)\})$. Let the regular map $\mathscr{F}: D_{2} \rightarrow C^{d}$ be given by $x \rightarrow$ $\left(g_{1}(x), g_{2}(x), \ldots, g_{d}(x)\right)$. We have $\partial / \partial x_{i}(\pi \circ \varphi)=\pi_{*}\left(\partial / \partial x_{i}\right)(\varphi)$ for $\varphi \in C\left(A_{C}\right)$.

Hence

$$
\frac{d}{d x}\left(\varphi^{\circ} F\right)=\frac{d}{d x}\left(\varphi^{\circ} \pi \circ \mathscr{F}\right)=\sum_{i=1}^{d} \frac{\partial\left(\varphi^{\circ} \pi\right)}{\partial x_{i}} g_{i}^{\prime}=\sum_{i=1}^{d} g_{i}^{\prime} \pi_{*} \frac{\partial}{\partial x_{i}}(\varphi) .
$$

Hence $l \delta(v)_{i}=\sum_{i=1}^{d} g_{i}^{\prime} X_{i}$ and $g_{i}^{\prime} \in K$. We have to check that $(\varphi \circ \pi \circ \mathscr{F}) \circ f$ is $\mathbf{Q}$-classical for $\varphi \in C\left(A_{C}\right)$. Let us put $G=\pi \circ \mathscr{F} \circ f: D_{1} \rightarrow D_{2} \rightarrow \mathbf{C}^{d} \rightarrow A_{C}$. It defines a $\mathbf{C}\left(D_{1}\right)$-rational point $w: \operatorname{Spec} \mathbf{C}\left(D_{1}\right) \rightarrow A_{\mathbf{C}\left(D_{1}\right)}$. As we have seen above $l \delta(w)=\sum_{i=1}^{d}\left(g_{1}(f)\right)^{\prime} \pi_{*}\left(\partial / \partial x_{i}\right)=\sum_{i=1}^{d} g_{i}(f)^{\prime} X_{i}$. Since $\left\{g_{i}(f)\right\}^{\prime}=g_{i}^{\prime}(f) f^{\prime}$ and since $g_{i}^{\prime} \in K,\left\{g_{i}(f)\right\}^{\prime}$ is $\mathbf{Q}$-classical. Let us put $\mathscr{K}=Q(x)\left\langle\left\{g_{i}(f)\right\}^{\prime}\right\rangle_{1 \leq i \leq d}$. Then by Lemma (3.5) there exist a point $P \in D_{1}$ and an extension $C$ of the field $C_{\mathscr{K}}$ of constants of $\mathscr{K}$ such that $C$ consists of classical numbers and finitely generated over $\mathbf{Q}(x)$, the $\left\{g_{i}(f)\right\}$ 's are regular at $P$ and such that $P$ is a $C$-valued point of $\left(D_{1}, \mathscr{K} C\right)$. We have $\varphi(G(P))=(\varphi \circ F)(f(P))$. By Theorem $(3.7) f(P)$ is a clas. sical number and hence $\varphi\left(F(f(P))=\left(\varphi^{\circ} F\right)(f(P))\right.$ is a classical number for any $\varphi \in C\left(A_{C}\right)$ if $F(P)$ is not a pole of $\varphi$ since $\varphi^{\circ} F$ is a $\mathbf{Q}$-classical function. Thus $\varphi^{\circ}$ $(\pi \circ \mathscr{F}) \circ f$ is $\mathbf{Q}$-classical for $\varphi \in C\left(A_{C}\right)$ by Proposition (3.10). This is what we had to prove.

COROLlary (3.18). If $a$ and $b$ are classical numbers, then $a^{b}$ is a classical number.

Proof. The function $e^{x}$ and $b \log x$ are $\mathbf{Q}$-classical by Examples (2.12) and (2.14). Hence the composite $e^{b \log x}$ is $\mathbf{Q}$-classical by Theorem (3.15). The value $e^{b \log a}$ of the $\mathbf{Q}$-classical function at the classical number $a$ is classical by Theorem (3.7) which is equal to $a^{b}$.

\section{\$4. Elementary functions}

J. Liouville [L] introduced the notion of elementary functions. All the functions are meromorphic over a domain $D$ of $\mathbf{C}$.

Definition (4.1). The field of elementary functions is generated by the following rules. 
(E1) Every polynomial $P(x) \in \mathbf{C}[x]$ is elementary.

(E2) If $f$ and $g$ are elementary functions, then the sum $f+g$, the difference $f-g$, the product $f g$ and the quotient $f / g$ (if $g \neq 0$ ) are elementary.

(E3) Let $a_{1}, a_{2}, \ldots, a_{n}$ be elementary functions. If a function $f$ satisfies an algebraic equation $f^{n}+a_{1} f^{n-1}+\cdots+a_{n}=0$, then $f$ is elementary.

(E4) If $f$ is elementary, then $\log f$ is elementary.

(E5) If $f$ is elementary, then $\exp f$ is elementary.

Since $\log x$ is not single valued, the operation (E4) should be clarified. We denote by $\log x$ any branch of $\log x=\log (1-(1-x))=1-x+\frac{(1-x)^{2}}{2}+$ $\cdots(|1-x|<1)$. If $f$ is holomorphic on $D \subset C$, we replace $D$ by a subdomain $D^{\prime}$ such that the branch $\log x$ is regular on $f\left(D^{\prime}\right)$. Then $\log f$ is composite $\log \circ f$ which is regular on $D^{\prime}$.

Remark (4.1.1). It follows from the nature of the operations (E3), (E4) and (E5) that if $f$ is an elementary function, then there exists almost a covering morphism $p: R \rightarrow \mathbf{P}_{\mathbf{C}}^{1}$ of Riemann surfaces such that $f$ is single valued and meromorphic over $R$.

EXAmples (4.2). The functions $\cos x=\left(e^{\imath x}+e^{-i x}\right) / 2, \sin x=\left(e^{i x}-e^{-i x}\right) /$ $2 i$ and $\arctan x=\frac{1}{2 i} \log \frac{i-x}{i+x}$ are elementary. By similar formulas, $\arcsin x$ and $\operatorname{arc} \cos x$ are elementary.

Lemma (4.3). If $f$ is elementary, then $f^{\prime}$ is elementary.

Proof. The lemma is proved by induction on the number of operations to obtain $f$ from $\mathbf{C}(x)$. For example if $a_{1}, a_{2}, \ldots, a_{n}$ are elementary functions, $f$ is algebraic over $\mathbf{C}\left(a_{1}, a_{2}, \ldots, a_{n}\right)$ and if $a_{1}^{\prime}, a_{2}^{\prime}, \ldots, a_{n}^{\prime}$ are elementary functions, then $f^{\prime}$ is in $\mathbf{C}\left(f, a_{1}, a_{2}, \ldots, a_{n}, a_{1}^{\prime}, a_{2}^{\prime}, \ldots, a_{n}^{\prime}\right)$ and hence elementary by (E2). Other cases are treated as easily as the above case and hence we omit the proof.

Proposition (4.4). An elementary function is classical.

Proof. Since $\log x$ and $\exp x$ are classical by Examples (2.12) and (2.14), the proposition follows from Definition (1.9) and Theorem (1.12) by induction, starting from $\mathbf{C}(x)$ on the number of times using the operations (E1), (E2), ..,(E5) to 
obtain the elementary function.

The set of elementary functions is closed under the composition.

Proposition (4.5). Let $f, g$ be elementary functions, then the composite function $g \circ f$ is elementary.

Proof. We prove the proposition by induction, starting from $\mathbf{C}(x)$ on the number of times using the operations (E1), (E2), ..,(E5) to obtain the function $g$. If $g \in \mathbf{C}(x)$, then $g \circ f$ is elementary by (E2). Let us assume that for any element of a field $K$ of elementary functions $h \circ f$ is elementary. Let $g$ be algebraic over $K$; $g^{n}+a_{1} g^{n-1}+\cdots+a_{n}=0$ with $a_{\imath} \in K, 1 \leq i \leq n$. Then $(g \circ f)^{n}+\left(a_{1} \circ f\right)(g \circ$ $f)^{n-1}+\cdots+\left(a_{n} \circ f\right)=0$. Since the $a_{i} \circ f$ 's are elementary by induction hypothesis, $g \circ f$ is elementary by (E2). If $g=\log h$ for $h \in K$, then $g \circ f=(\log h) \circ f=$ $\log (h \circ f)$ is elementary by induction hypothesis and (E4). If $g=\exp h$ for $h \in K$, then $g \circ f=(\exp h) \circ f=\exp (h \circ f)$ is elementary by induction hypothesis and (E5).

In the definition of elementary function, it is natural to start from $\mathbf{Q}(x)$.

Definition (4.6). The field of $\mathbf{Q}$-elementary functions is generated from $\mathbf{Q}(x)$ by the following rules.

(F1) Any polynomial $P(x) \in \mathbf{Q}[x]$ is $\mathbf{Q}$-elementary.

(F2) If $f$ and $g$ are $\mathbf{Q}$-elementary functions, then the sum $f+g$, the difference $f-g$, the product $f g$ and the quotient $f / g($ if $g \neq 0$ ) are Q-elementary.

(F3) Let $a_{1}, a_{2}, \ldots, a_{n}$ be $\mathbf{Q}$-elementary functions. If a function $f$ satisfies an algebraic equation $f^{n}+a_{1} f^{n-1}+\cdots+a_{n}=0$, then $f$ is Q-elementary.

(E4) If $f$ is $\mathbf{Q}$-elementary, then $\log f$ is $\mathbf{Q}$-elementary.

(E5) If $f$ is $\mathbf{Q}$-elementary, then $\exp f$ is $\mathbf{Q}$-elementary.

A constant $\mathbf{Q}$-elementary function is called an elementary number. The field of $\mathbf{Q}$-elementary numbers is denoted by $\mathbf{Q}^{\mathrm{cl}}$.

Example (4.7). The functions in Example (4.2) are $\mathbf{Q}$-elementary. $e=$ $\exp (1)$ is an elementary number since $\exp x$ is $\mathbf{Q}$-elementary and 1 is an elementary number. Every algebraic number is elementary by (F3). Let $f_{0}(x)$ and $f_{1}(x)$ be branches of $\log x$ regular around 1 with $f_{0}(1)=0, f_{1}(1)=2 \pi i$. Then $\left(f_{0}-f_{1}\right)(1)=-2 \pi i$ is elementary and hence $\pi$ is an elementary number. 
The argument of Lemma (4.1) allows us to prove the following.

Proposition (4.8). If $f$ is a $\mathbf{Q}$-elementary function, then $f^{\prime}$ is a $\mathbf{Q}$-elementary function.

The following proposition is proved by the same method as Proposition (4.5).

Proposition (4.9). If $f$ and $g$ are $\mathbf{Q}$-elementary, then the composite function $g \circ f$ is $\mathbf{Q}$-elementary.

Theorem (4.10). A $\mathbf{Q}$-elementary function is $\mathbf{Q}$-classical.

Proof. This follows from Theorem (3.15) by induction.

In particular we have

Corollary (4.11). The field $\mathbf{Q}^{\mathrm{el}}$ is a subfield of $\mathbf{Q}^{\mathrm{cl}}$.

The following natural question arises.

PROBLEM (4.12). Is the field $\mathbf{Q}^{\mathrm{cl}}$ a proper subfield of $\mathbf{Q}^{\mathrm{cl}}$ ? If so, contruct an example.

One can guess that an elliptic integral may give an example.

\section{\$5. Periods of algebraic varieties defined over $\mathbf{Q}^{\mathrm{cl}}$}

Let $K$ be an algebraic number field and $C$ be an elliptic curve defined over $K$. Replacing $K$ by a finite algebraic extensions, we may assume that there exist a $K$-morphism $f: C \rightarrow \mathbf{P}_{K}^{1}$ of degree 2. Let $\omega$ be a non-zero regular 1 -form over $C ; \omega \in H^{0}\left(C, \Omega_{C / K}^{1}\right)$. If $\omega^{\prime} \in H^{0}\left(C, \Omega_{C / K}^{1}\right)$, then $\omega^{\prime}=\lambda \omega$ for some $\lambda \in K$ since $H^{0}\left(C, \Omega_{C / K}^{1}\right) \simeq K$. Let $\gamma \in H_{1}\left(C \otimes_{K} \mathbf{C}, \mathbf{Q}\right)$. We call $\int_{\gamma} \omega$ a period of $C$ over $K$.

Proposition (5.1). Every period of an elliptic curve defined over an algebraic number field is classical.

Proof. We have $\overline{\mathbf{Q}}$-morphism $f: C_{\overline{\mathbf{Q}}} \rightarrow \mathbf{P}_{\overline{\mathbf{Q}}}^{1}$ of algebraic curves. The set of 
$\overline{\mathbf{Q}}$-valued points of $\left(\mathbf{P}_{\mathbf{C}}^{1}, \mathbf{Q}(x)\right)$ are dense on $\mathbf{P}_{\mathbf{C}}$. Therefore $\int_{\gamma} \boldsymbol{\omega}$ is expressed by a finite sum of integrals $(1 \leq i \leq n)$ on an open set of $\mathbf{P}_{\mathbf{C}}^{1}$.

$$
\int_{P_{i}}^{Q_{i}} h(x) d x
$$

where $P_{i}, Q_{i}$ are $\mathbf{Q}$-valued points of $\left(\mathbf{P}_{\mathbf{C}}^{1}, \mathbf{Q}(x)\right)$ and $h(x)$ is an algebraic function in $\overline{\mathbf{Q}}\left(C_{\overline{\mathbf{Q}}}\right)$. Since $h(x)$ is $\mathbf{Q}$-classical, the above integral

$$
\int_{P_{i}}^{Q_{i}} h(x) d x
$$

is classical by Theorem (3.8).

The above argument shows

Proposition (5.2). Let $C$ be an elliptic curve defined over a field $K$ of classical numbers. Then every period of $C$ defined over $K$ is classical.

Let now $C$ be a smooth projective curve defined over a field $K$. Let $\gamma$ be a cycle on $C \otimes_{K} \mathbf{C}$. We call $\int_{\gamma} \omega$ a period of $C$ over $K$. The above argument gives us.

Proposition (5.3). Let $C$ be a curve defined over a field $K$ of classical numbers. Then every period of $C$ defined over $K$ is classical.

The most general form of this type of result is as follows.

THEOREM (5.4). Let $V$ be a complete and non-singular algebraic variety defined over a field of classical numbers. Let $\omega \in H^{0}\left(V, \Omega_{V / K}^{1}\right)$ and $\gamma \in H_{1}\left(V \otimes_{K} \mathbf{C}, \mathbf{Q}\right)$. Then the integral $\int_{\gamma} \omega$ is a classical number.

Proof. We may replace the field $K$ by a finite algebraic extension by Corollary (3.3). Therefore by Chow's Theorem there exist a non-singular projective variety $\tilde{V}$ obtained from $V$ by a successive blowing-up $p: \tilde{V} \rightarrow V$. The variety $\tilde{V}$ and the morphism $p$ are defined over $K$. We know that the morphism $p$ induces an isomorphisms $p^{*}: H^{0}\left(V_{\mathbf{C}}, \Omega_{V_{\mathbf{C}}}^{1}\right) \simeq H^{0}\left(\tilde{V}_{\mathbf{C}}, \Omega_{V_{\mathbf{C}}}^{1}\right)$ and $p_{*}: H_{1}\left(\tilde{V}_{\mathbf{C}}, \mathbf{Q}\right) \simeq H_{1}\left(V_{\mathbf{C}}\right.$, 
Q). Therefore there exists a 1 -cycle $\tilde{\gamma}$ on $\tilde{V}_{C}$ such that $p_{*} \tilde{\gamma}=\gamma$. Then $\int_{\tilde{r}} p^{*} \omega=$ $\int_{p_{*} \tilde{r}} \omega=\int_{\gamma} \omega$. Therefore we may assume that $V$ is projective. then by a theorem of Lefschetz there exists a smooth projective curve $C$ on $V$ defined over a finite algebraic extension of $K$ such that the morphism $i_{*}: H_{1}\left(C_{\mathbf{C}}, \mathbf{Q}\right) \rightarrow H_{1}\left(V_{\mathbf{C}}\right.$, Q) induced by the inclusion $i: C \subset V$ is surjective. Therefore there exist a 1 -cycle $\delta \in H_{1}\left(C_{\mathbf{C}}, Z\right)$ such that $i_{*} \delta=\gamma$. Then $\int_{\gamma} \omega=\int_{i_{*} \delta} \omega=\int_{\gamma} i^{*} \omega$. The latter is a classical number by the argument of the proof of Proposition (5.1) and the theorem is proved.

\section{§6. Examples}

Example (6.1). The hypergeometric function.

The hypergeometric series $F(a, b ; c ; x)$ is defined by

$$
\begin{aligned}
& F(a, b ; c ; x)=1+\frac{a \cdot b}{1 . c} x+ \frac{a(a+1) b(b+1)}{1.2 \cdot c(c+1)} x^{2}+ \\
& \frac{a(a+1)(a+2) b(b+1)(b+2)}{1.2 .3 . c(c+1)(c+2)} x^{3}+\cdots .
\end{aligned}
$$

We know that the series is convergent and defines an analytic function for $|x|<1$ (cf. [WW], Chap. XIV). The function $F(a, b ; c ; x)$ is a solution of the hypergeometric differential equation

$$
x(1-x) \frac{d^{2} y}{d x^{2}}+\{c-(a+b+1) x\} \frac{d y}{d x}-a b y=0 .
$$

We show that the hypergeometric function $F(a, b ; c ; x)$ is $\mathbf{Q}$-classical as a function of $x$ when $a, b, c \in \mathbf{Q}$ with $b \geq 1, c-b \geq 1$. In fact we have

$$
F(a, b ; c ; x)=\frac{\Gamma(c)}{\Gamma(b) \Gamma(c-b)} \int_{0}^{1} u^{b-1}(1-u)^{c-b-1}(1-u x)^{-a} d u
$$

by [WW], Chap. XIV, 14.6 Example 1. It is sufficient to show by Corollary (3.11) that the initial conditions of $F(a, b ; c ; x)$ at a $\mathbf{Q}(\sqrt{-1})$-valued point $\sqrt{-1}$ of $(\mathbf{C}, \mathbf{Q}(\sqrt{-1})(x))$ are classical: namely $F(a, b ; c ; \sqrt{-1}), F^{\prime}(a, b ; c ; \sqrt{-\dot{1}})$ are classical numbers. The integrand $u^{b-1}(1-u)^{c-b-1}(1-u x)^{-a}$ as a function of $u$ is $\mathbf{Q}$-classical by Proposition (3.12) and hence by Theorem (3.8) and Example (3.9.2), the initial conditions 


$$
F(a, b ; c ; \sqrt{-1})=\int_{0}^{1} u^{b-1}(1-u)^{c-b-1}(1-\sqrt{-1} u)^{-a} d u
$$

and

$$
F^{\prime}(a, b ; c ; \sqrt{-1})=\int_{0}^{1}\left\{-\sqrt{-1} a u^{b}(1-u)^{c-b-1}(1-\sqrt{-1} u)^{-a-1}\right\} d u
$$

are classical numbers.

It is natural to ask the following question.

Problem (6.4). If $a, b, c$ are classical, is the hypergeometric function $F(a, b$; $c ; x) \mathbf{Q}$-classical?

Example (6.5). The Weierstrass functions.

Let $L \subset \mathbf{C}$ be a lattice. We have seen in Example (2.15) that if $g_{2}(L)$ and $g_{3}(L)$ are rational numbers, then $\wp(x ; L)$ is $\mathbf{Q}$-classical. The same argument shows that $\wp(x ; L)$ is $\mathbf{Q}$-classical if $g_{2}(L)$ and $g_{3}(L)$ are classical. The function $\zeta(x)=\zeta(x ; L)$ is defined by

$$
\zeta(x)-x^{-1}=-\int_{0}^{x}\left\{\wp(x)-x^{-2}\right\} d x
$$

Since $0 \in \mathbf{C}$ is a $\mathbf{Q}\left(g_{2}, g_{3}\right)$-valued point of $\left(\mathbf{C}, \mathbf{Q}\left(x, \wp^{\prime}(x), \boldsymbol{\gamma}^{\prime}(x)\right)\right.$ by Lemma (2.4), $\zeta(x)-x^{-1}$ is $\mathbf{Q}$-classical and hence $\zeta(x)$ is $\mathbf{Q}$-classical if $g_{2}(L)$ and $g_{3}(L)$ are rational. More generally $\zeta(x)$ is $Q$-classical if $g_{2}(L)$ and $g_{3}(L)$ are classical. The function $\sigma(x)$ is defined by the differential equation

$$
\sigma^{\prime}(x) / \sigma(x)=\zeta(x)
$$

coupled with the condition $\sigma(x) / x$ is regular at $x=0$ and takes the value 1 . Namely

$$
\frac{(\sigma(x) / x)^{\prime}}{(\sigma(x) / x)}=\zeta(x)-\frac{1}{x} .
$$

Since $0 \in \mathbf{C}$ is a $\boldsymbol{Q}\left(g_{2}(L), g_{3}(L)\right)$-rational point of $\left(\mathbf{C}, \mathbf{Q}\left(g_{2}(L), g_{3}(L)\right)<x\right.$, $\zeta(x)\rangle)$, so $\sigma(x) / x$ and consequently $\sigma(x)$ are $\mathbf{Q}$-classical if $g_{2}(L)$ and $g_{3}(L)$ are classical numbers. $\left(\mathbf{Q}\left(g_{2}(L), g_{3}(L)\right)\langle x, \zeta(x)\rangle\right.$ denotes the differential field generated by $x$ and $\zeta(x)$ over $\mathbf{Q}\left(g_{2}(L), g_{3}(L)\right)$. 
More generally we can prove the following result.

Proposition (6.5.1). The following conditions are equivalent.

(1) $g_{2}(L)$ and $g_{3}(L)$ are classical numbers.

(2) $\boldsymbol{\wp}(x ; L)$ is $\mathbf{Q}$-classical.

(3) $\sigma(x ; L)$ is $\mathbf{Q}$-classical.

(4) $\sigma(x ; L), \zeta(x ; L)$ and $\wp(x ; L)$ are $\mathbf{Q}$-classical.

Proof. The equivalence of the conditions (2), (3) and (4) is proved by the above argument. As in Example (2.15) we can prove that the condition (1) implies the condition (2). Let us prove that the condition (2) implies the condition (1). If $\boldsymbol{\gamma}(x ; L)$ is $\mathbf{Q}$-classical, then $\boldsymbol{\gamma}^{\prime}(x ; L)$ is $\mathbf{Q}$-classical too. We have an algebraic differential equation

$$
\wp^{\prime}(x ; L)^{2}=4 \wp(x ; L)^{3}-g_{2}(L) \wp(x ; L)-g_{3}(L) .
$$

We can find two rational numbers $a, b$ such that $\wp(x ; L)$ is regular at the points $a$ and $b$ and such that $\wp(a ; L) \neq \wp(b ; L)$ or equivalently

$$
\left|\begin{array}{ll}
\wp(a ; L) & 1 \\
\wp(b ; L) & 1
\end{array}\right| \neq 0 \text {. }
$$

It follows from (6.5.2)

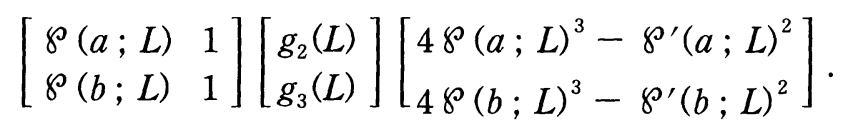

$\gamma^{\prime}(a ; L), \boldsymbol{\gamma}^{\prime}(a ; L), \wp^{\prime}(b ; L)$ and $\boldsymbol{\wp}^{\prime}(b ; L)$ are classical by Theorem (3.7). Hence (6.5.4) is a linear equation for $g_{2}(L)$ and $g_{3}(L)$ with coefficients in $\mathbf{Q}^{\mathrm{cl}}$. By condition (6.5.3), we can solve this linear equation so that $g_{2}(L), g_{3}(L) \in \mathbf{Q}^{\mathrm{cl}}$.

EXAmple (6.6). The Legendre functions.

For $n \in \mathrm{C}$, the Legendre function $P_{n}(x)$ is defined by

$$
P_{n}(x)=\frac{1}{2 \pi i} \int_{C} \frac{\left(t^{2}-1\right)^{n}}{2^{n}(t-x)^{n+1}} d t
$$

where $C$ is a suitable contour (cf. [WW] Chap. XV, 15.2, p. 302-307). The Legendre function $P_{n}(x)$ satisfies the differential equation

$$
\left(1-x^{2}\right) d^{2} P_{n} / d x^{2}-2 x d P_{n} / d x+n(n+1) P_{n}=0 .
$$

Therefore if $n$ is a classical number, the Legendre function $P_{n}(x)$ is $\mathbf{Q}$-classical 
by Corollary (3.11). The Legendre function $Q_{n}(x)$ of the second kind is defined by

$$
Q_{n}(x)=\frac{1}{4 i \sin n \pi} \int_{D} \frac{\left(t^{2}-1\right)^{n}}{2^{n}(t-x)^{n+1}} d t
$$

where $D$ is a suitable contour. The Legendre function $Q_{n}(x)$ of second kind satisfies the differential equation (6.7) (cf. [WW], Chap. XV, 15.3, p. 316). By a similar argument, we can prove that the Legendre function $Q_{n}(x)$ of the second kind is $Q$-classical if $n$ is a classical number.

Example (6.8). The Bessel functions.

For an integer $n$, the Bessel function $J_{n}(x)$ is defined by the formula

$$
J_{n}(x)=\frac{1}{2 \pi i}\left(\frac{1}{2} x\right)^{n} \int^{(0+)} t^{-n-1} \exp \left(t-\frac{x^{2}}{4 t}\right) d t
$$

where the contour encircles the origin once counter-clockwise. The Bessel function $J_{n}(x)$ is a solution of the linear differential equation

$$
d^{2} y / d x^{2}+(1 / x) d y / d x+\left(1-n^{2} / x^{2}\right) y=0 .
$$

Therefore the Bessel function $J_{n}(x)$ is $\mathbf{Q}$-classical (if $n$ is an integer) by the argument of Example (6.1). For a complex number the Bessel function $J_{n}(x)$ is defined by the equation

$$
J_{n}(x)=\frac{x^{n}}{2^{n+1} \pi i} \int_{-\infty}^{(0+)} t^{-n-1} \exp \left(t-\frac{x^{2}}{4 t}\right) d t .
$$

The Bessel function $J_{n}(x)$ is a solution of the linear differential equation (6.9) ([WW], Chap. XVII, 17.2). It is natural to ask the following question.

Problem (6.10). If $n$ is a classical number, the Bessel function $J_{n}(x)$ is Q-classical?

Example (6.11). The Jacobian elliptic functions.

For a complex number $\tau$ with $\operatorname{Im} \tau>0$, we write $q=e^{\pi i \tau}$. The following four types of theta functions are introduced. First $\theta_{4}(x, \tau)$ is defined by the equation

$$
\theta_{4}(x, \tau)=\sum_{n \in \mathbf{Z}}(-1)^{n} q^{n^{2}} e^{2 \pi n i x}
$$

We put 


$$
\begin{aligned}
& \theta_{3}(x, \tau)=\theta_{4}\left(x+\frac{1}{2} \pi, \tau\right) \\
& \theta_{1}(x, \tau)=-i e^{i x+\frac{1}{4} \pi i \tau} \theta_{4}\left(x+\frac{1}{2} \pi \tau, \tau\right) \text { and } \\
& \theta_{2}(x, \tau)=\theta_{1}\left(x+\frac{1}{2} \pi, \tau\right)
\end{aligned}
$$

We sometimes omit $\tau$ and write $\theta_{i}(x, \tau)=\theta_{i}(x)$ for $1 \leq i \leq 4$. The relations with the theta functions with characteristic defined for example in [M1] are

$$
\begin{aligned}
& \theta_{1}(\pi x, \tau)=\theta_{11}(x, \tau), \\
& \theta_{2}(\pi x, \tau)=\theta_{10}(x, \tau), \\
& \theta_{3}(\pi x, \tau)=\theta_{00}(x, \tau), \\
& \theta_{4}(\pi x, \tau)=\theta_{01}(x, \tau) .
\end{aligned}
$$

We show that $\theta_{1}(x, \tau), \theta_{2}(x, \tau), \theta_{3}(x, \tau), \theta_{4}(x, \tau)$ are $\mathbf{Q}$-classical if and only if the theta constants $\theta_{2}=\theta_{2}(0, \tau), \theta_{3}=\theta(0, \tau), \theta_{4}=\theta_{4}(0, \tau)$ are classical $\left(\theta_{1}=\theta_{1}(0, \tau)=0\right)$. Let us sketch the proof of this assertion. The only if part follows from Theorem (3.7). Let us prove the if part. Let us put $k^{1 / 2}=$ $\theta_{2}(0, \tau) / \theta_{3}(0, \tau)$ and consider an elliptic curve $w^{2}=\left(1-u^{2}\right)\left(1-k^{2} u^{2}\right)$. If we put $y=\frac{\theta_{3} \theta_{1}\left(x / \theta_{3}^{2}\right)}{\theta_{2} \theta_{4}\left(x / \theta_{3}^{2}\right)}$. Differentiating the addition theorem and using Jacobi's derivative formula, we conclude that $y$ satisfies the differential equation

$$
(d y / d x)^{2}=\left(1-y^{2}\right)\left(1-k^{2} y^{2}\right)
$$

(cf. [WW], Chap. XXI, 21.6).

The argument in Example (2.15) shows that

$y=\frac{\theta_{3} \theta_{1}\left(x / \theta_{3}^{2}\right)}{\theta_{2} \theta_{4}\left(x / \theta_{3}^{2}\right)}$ is $\mathbf{Q}$-classical. We denote $y=\frac{\theta_{3} \theta_{1}\left(x / \theta_{3}^{2}\right)}{\theta_{2} \theta_{4}\left(x / \theta_{3}^{2}\right)}$ by sn $x$ according to Jacobi. We set

$$
\begin{aligned}
& \text { cn } x=\frac{\theta_{4} \theta_{2}\left(x / \theta_{3}^{2}\right)}{\theta_{2} \theta_{4}\left(x / \theta_{3}^{2}\right)}, \\
& \text { dn } x=\frac{\theta_{4} \theta_{3}\left(x / \theta_{3}^{2}\right)}{\theta_{2} \theta_{4}\left(x / \theta_{3}^{2}\right)} .
\end{aligned}
$$

Then we have

$$
\begin{gathered}
\operatorname{sn}^{2} x+\operatorname{cn}^{2} x=1, \\
k^{2} \operatorname{sn}^{2} x+\operatorname{dn}^{2} x=1
\end{gathered}
$$


(cf. [WW], Chap. XXII, 22.11).

Hence $\operatorname{cn} x$ and $\operatorname{dn} x$ are $\mathbf{Q}$-classical by Proposition (3.12). We write $K=$ $\int_{0}^{1}\left(1-t^{2}\right)^{-1 / 2}\left(1-k^{2} t^{2}\right)^{-1 / 2} d t$ which is classical by Theorem (3.8). Since we have the equations

$$
\begin{aligned}
& \frac{d \operatorname{sn} x}{d x}=\operatorname{cn} x \operatorname{dn} x \\
& \frac{d \operatorname{cn} x}{d x}=-\operatorname{sn} x \operatorname{dn} x \\
& \frac{d \operatorname{dn} x}{d x}=-k^{2} \operatorname{sn} x \operatorname{cn} x
\end{aligned}
$$

$K=\mathbf{Q}\left(\theta_{2}, \theta_{3}, \theta_{4}\right)(x, \operatorname{cn} x, \operatorname{sn} x, \operatorname{dn} x)$ is a differential field (see [WW], Chap. XXII, 22.12) and $0 \in \mathbf{C}$ is a $Q\left(\theta_{1}, \theta_{2}, \theta_{3}\right)$-valued point of $(\mathbf{C}, K)$ by the argument of Lemma (3.4). Hence the function $E(x)=\int_{0}^{x} \mathrm{dn}^{2} x d x$ is $\mathbf{Q}$-classical. We set $K=\frac{\pi}{2} \theta_{3}^{2}$ and $E=E(K)$. Then $K$ is classical by assumption and by Example (2.14) and hence $E(K)$ is classical by Theorem (3.7). If we put $\Theta(x)=$ $\theta_{4}\left(x \theta_{3}^{-2}\right)$ and $Z(x)=\Theta^{\prime}(x) / \Theta(x)$, then we know $Z(x)=E(x)-x E / K$ and $\theta_{4}\left(x \theta_{3}^{-2}\right)=\theta_{4} \int_{0}^{x} Z(x) d x$ by [WW], Chap. XXII, 22.731. Therefore $Z(x)$ is $\mathbf{Q}$-classical and hence $\theta_{4}\left(x \theta_{3}^{-2}\right)$ is $\mathbf{Q}$-classical and consequently $\theta_{3}\left(x \theta_{3}^{-2}\right)$, $\theta_{1}\left(x \theta_{3}^{-2}\right), \theta_{2}\left(x \theta_{3}^{-2}\right)$ are $\mathbf{Q}$-classical since we have seen $\operatorname{sn} x, \mathrm{dn} x, \operatorname{cn} x$ are $\mathbf{Q}$-classical. Since we assume that the theta constant $\theta_{3}$ is classical, by Theorem (3.15) $\theta_{1}(x), \theta_{2}(x), \theta_{3}(x), \theta_{4}(x)$ are $\mathbf{Q}$-classical.

It is natural to expect a generalization of what is proved above.

Problem (6.12). Let $S \subset \mathbf{R}^{2}$ be a carefully chosen set of theta characteristics so that $x \rightarrow\left(\theta_{m}(x, \tau)\right)_{m \in S} \in \mathbf{P}^{N}(N=|s|-1)$ gives an embedding of elliptic curve $\mathbf{C} /(\mathbf{Z}+\mathbf{Z} \tau)$. Then the $\theta_{m}(x, \tau)$ 's are $\mathbf{Q}$-classical if and only if the theta constants $\theta_{m}(0, \tau)$ are classical?

Example (6.13). The Gamma function.

Gauss defines the function $\Pi(x)$ by

$$
\Pi(x)=\lim _{k \rightarrow \infty} \frac{1.2 .3 \ldots k}{(x+1)(x+2) \ldots(x+k)} k^{x}
$$


and shows that $\Pi(x)=\int_{0}^{\infty} e^{-t} t^{x} d t . \Pi(x)$ is a meromorphic function over $\mathbf{C}$ with poles at $\mathbf{Z}_{-}=\{n \in \mathbf{Z} \mid n<0\}$. We have

$$
\Pi(x+1)=(x+1) \Pi(x)
$$

and $\Pi(0)=1$. It follows $\Pi(x)=x$ ! for a positive integer $x$. We notice that the Gamma function introduced in [W.W] is equal to our $\Pi(x-1)$. The notation of [W.W] is standard but we use the notation of Gauss since we owe him much. It is well known that the function $\Pi(x)$ satisfies no algebraic differential equation over $\mathbf{C}(x)$ or $\Pi(x), \Pi^{\prime}(x), \Pi^{(2)}(x), \ldots$ are algebraically independent over $\mathbf{C}(x)$. In particular $\Pi(x)$ is not classical. The value $\Pi(r)$ of the Gamma function is, however, classical for any rational number $r$ if $x=r$ is not a pole of $\Pi(x)$. In fact $\Pi(r)$ is related with periods of algebraic curves. The following marvelous idea is due to Gauss [G].

He proved in article 24 of [G]

$$
F(a, b, c ; 1)=\frac{\Pi(c-1) \Pi(c-a-b-1)}{\Pi(c-a-1) \Pi(c-b-1)}
$$

if the real part $R(c-a-b)>0$.

On the other hand it is easy to see

$$
t=\sin t . F\left(\frac{1}{2}, \frac{1}{2}, \frac{3}{2} ; \sin ^{2} t\right) .
$$

Substituting $t=\frac{1}{2} \pi$ in (6.13.3), we get

$$
\frac{1}{2} \pi=F\left(\frac{1}{2}, \frac{1}{2}, \frac{3}{2} ; \frac{1}{2}\right)=\frac{\Pi(1 / 2) \Pi(-1 / 2)}{\Pi(0) \Pi(0)} .
$$

Since $\Pi\left(\frac{1}{2}\right)=\frac{1}{2} \Pi\left(-\frac{1}{2}\right)$ by (6.13.1), we get $\pi=\Pi\left(-\frac{1}{2}\right)^{2}$ and hence

$$
\Pi\left(-\frac{1}{2}\right)=\sqrt{\pi} \text { and } \Pi\left(\frac{1}{2}\right)=\frac{1}{2} \sqrt{\pi}
$$

Substituting $t=\frac{1}{2} \pi$ in a similar formula

$$
\sin n t=n \sin t . F\left(\frac{1}{2} n+\frac{1}{2},-\frac{1}{2} n+\frac{1}{2}, \frac{3}{2}, \sin ^{2} t\right)
$$


which is also easy to see, we get

$$
\sin \frac{n \pi}{2}=\frac{n \Pi(1 / 2) \Pi(-1 / 2)}{\Pi(-n / 2) \Pi(n / 2)} .
$$

If we put $n=2 x$, then it follows from (6.13.4) and (6.13.5),

$$
\begin{gathered}
\Pi(-x) \Pi(x)=\frac{x \pi}{\sin x \pi}, \\
\Pi(-x) \Pi(x-1)=\frac{\pi}{\sin x \pi} .
\end{gathered}
$$

Now let us show how $\Pi\left(\frac{1}{4}\right)$ is related with the elliptic integral $\int_{0}^{1} \frac{d x}{\left(1-x^{4}\right)^{1 / 2}}=A$ which is a classical number by Theorem (3.8). Let $\lambda, \mu$ be positive numbers. Then

$$
\begin{aligned}
\int_{0}^{x} x^{\lambda-1}\left(1-x^{\mu}\right)^{\nu} d x & =\frac{x^{\lambda}}{\lambda}-\frac{\nu x^{\mu+\lambda}}{\mu+\lambda}+\frac{\nu(\nu-1) x^{2 \mu+\nu}}{1.2 .(2 \mu+\lambda)}-\cdots \\
& =\frac{x^{\lambda}}{\lambda} F\left(-\nu, \frac{\lambda}{\mu}, \frac{\lambda}{\mu}+1, x^{\mu}\right) .
\end{aligned}
$$

We evaluating (6.13.8) at $x=1$, it follows, from (6.13.2)

$$
\int_{0}^{1} x^{\lambda-1}\left(1-x^{\mu}\right)^{\nu} d x=\frac{\Pi(\lambda / \mu) \Pi(\nu)}{\lambda \Pi(\lambda / \mu+\nu)} .
$$

It follows from (6.13.9) for $\lambda=1, \mu=4, \nu=-\frac{1}{2}$,

$$
A=\frac{\Pi(1 / 4) \Pi(-1 / 2)}{\Pi(-1 / 4)} \text {. }
$$

Since $\Pi\left(\frac{1}{4}\right) \Pi\left(-\frac{1}{4}\right)=\frac{\pi / 4}{\sin \pi / 4}=\pi / \sqrt{8}$ by (6.13.6), we get by (6.13.4) and (6.13.10)

$$
\Pi\left(\frac{1}{4}\right)=\left(\frac{1}{8} \pi A^{2}\right)^{1 / 4}, \quad \Pi\left(-\frac{1}{4}\right)=\left[\pi^{3} /\left(8 A^{2}\right)\right]^{1 / 4} .
$$

Therefore $\Pi\left(\frac{1}{4}\right)$ and $\Pi\left(-\frac{1}{4}\right)$ are classical numbers. Now it follows from (6.13.2) and (6.13.4) that $\Pi\left(\frac{n}{4}\right)$ is classical for $n \in \mathbf{Z}$ unless $\frac{n}{4}$ is a negative integer. 
Next we show how $\Pi\left(-\frac{1}{5}\right), \Pi\left(-\frac{2}{5}\right), \Pi\left(-\frac{3}{5}\right)$ and $\Pi\left(-\frac{4}{5}\right)$ are related with periods of an algebraic curve; we set

$$
\begin{aligned}
& \int_{0}^{1} \frac{d x}{\left(1-x^{5}\right)^{1 / 5}}=C, \int_{0}^{1} \frac{d x}{\left(1-x^{5}\right)^{2 / 5}}=D, \int_{0}^{1} \frac{d x}{\left(1-x^{5}\right)^{3 / 5}}=E, \\
& \int_{0}^{1} \frac{d x}{\left(1-x^{5}\right)^{4 / 5}}=F . \\
\text { Since } & \frac{d x}{\left(1-x^{5}\right)^{1 / 5}}, \frac{d x}{\left(1-x^{5}\right)^{2 / 5}}, \frac{d x}{\left(1-x^{5}\right)^{3 / 5}} \text { and } \frac{d x}{\left(1-x^{5}\right)^{4 / 5}} \text { are regular }
\end{aligned}
$$
differential of the first kind on the compactification of an algebraic curve $p: R \rightarrow$ $\mathbf{C}$, where $R=\left\{(x, y) \in \mathbf{C}^{2} \mid y^{5}=1-x^{5}\right\}$ and $p(x, y)=x$ by definition, $C, D$, $E, F$ are classical numbers by Theorem (3.8). It follows from (6.13.2) and (6.13.8)

$$
\begin{aligned}
& C=\Pi(1 / 5) \Pi(-1 / 5), D=\frac{\Pi(1 / 5) \Pi(-3 / 5)}{\Pi(-1 / 5)}, E=\frac{\Pi(1 / 5) \Pi(-3 / 5)}{\Pi(-2 / 5)}, \\
& F=\frac{\Pi(1 / 5) \Pi(-4 / 5)}{\Pi(-3 / 5)} .
\end{aligned}
$$

By (6.13.1) $\Pi\left(\frac{1}{5}\right)=\frac{1}{5} \Pi\left(-\frac{1}{5}\right)$ and hence we have

$$
\begin{aligned}
& \Pi(-1 / 5)=\left[5 C^{4}(D E F)\right]^{1 / 5}, \Pi(-2 / 5)=\left[25 C^{3} D^{3} /\left(E^{2} F^{2}\right)^{1 / 5}, \Pi(-3 / 5)=\right. \\
& {\left[125 C^{2} D^{2} E^{2} / F^{3}\right]^{1 / 5}, \Pi(-4 / 5)=(625 C D E F)^{1 / 5} .}
\end{aligned}
$$

It follows from (6.13.1) that $\Pi\left(\frac{n}{5}\right)$ is classical for $n \in \mathbf{Z}$ except for $n=-5$, $-10,-15, \ldots$, where $\Pi(x)$ has poles. For the same reason $\Pi(r)$ is classical for a rational number $r$ unless $r$ is a negative integer.

Problem (6.14). For a classical number $r$ not equal to a negative integer, is $\Pi(r)$ classical?

The logarithmic derivative $\Pi^{\prime}(x) / \Pi(x)$, which we denote by $\Psi(x)$, is also an interesting function. The value $\Psi(0)$ is the minus of Euler's constant:

$$
-\Psi(0)=\lim _{n \rightarrow \infty}\left(\sum_{k=1}^{n} 1 / k-\log n\right) .
$$

(cf. Article 30, Gauss [G]). 
It is natural to ask

QUESTION (6.15.1). Is Euler's constant classical?

Or more generally

QUESTION (6.15.2). Is $\Psi(r)$ classical for a rational number $r(r \neq-1$, $-2, \ldots)$ ?

Gauss proved that for integers $m, n$ with $0 \leq n<m, \Psi(-n / m)$ is a Q-linear combination of

$\pi \cot (l m / n), \log 2, \log n$ and $\cos (l m / n) \log (2-2 \cos (l \pi / n))$,

$l$ being integers satisfying $1 \leq l \leq n-1$. So it follows from the well-known formula

$$
\Psi(x+1)=\Psi(x)+1 /(1+x)
$$

and Theorem (3.7) that Question (6.15.2) is reduced to Question (6.15.1).

\section{\$7. Concluding remarks}

We can dispense with condition (A.3) of Definition (2.4). In fact this condition is superfluous. Dropping this condition from Definition (2.4), we can arrive at really a natural definition of $\mathbf{Q}$-classical functions. Let us call thus obtained classical functions generalized $\mathbf{Q}$-classical functions. All the results of this paper remain valid for generalized $\mathbf{Q}$-classical functions. But their proofs are rather messy and if we present the whole theory in logical order, it would not be comprehensive.

For this reason we want to clarify only two crucial points. In the course of the explanation, disadvantage of arranging the theorems in logical order will be apparent. Let us assume that the morphism $r: S^{\prime} \rightarrow \mathbf{P}_{\mathbf{C}}^{1}$ is ramified at the point $P \in S^{\prime}$ in Definition (2.4). We argue for simplicity under the hypothesis that $r(P)$ is not the point at infinity. We may replace $S^{\prime}$ by a disc $D$ on $S^{\prime}$ centered at $P$ so that $r: S^{\prime}=D \rightarrow \mathbf{C} \subset \mathbf{P}_{\mathbf{C}}^{1}$ is defined by $z \rightarrow z^{r}=x-r(P), z$ being the coordinate on the disc $D$. The number $r(P)$ is a classical number and $x-r(P)$ is a $\mathbf{Q}$-classical function since $P$ is a $C_{\mathscr{K}}$-valued point of $(D, \mathscr{K})$ and $\mathbf{Q}(x) \subset \mathscr{K}$. The function $\boldsymbol{z}$ is a $\mathbf{Q}$-classical function by Proposition (3.12) for $\mathbf{Q}$-classical function. In other words we have to use Proposition (3.12) and it should be 
proved beforehand.

Now Lemma (2.3) holds if we replace $\mathbf{Q}(x)$ by $\mathbf{Q}(x, z)$ and $x-\boldsymbol{a}$ by $z$. Namely we consider Laurent expansion with respect to $\boldsymbol{z}$.

Now that Lemma (2.3) is modified, generalized Lemma (2.6) follows under the assumption $\mathbf{Q}(x, z) \subset \mathscr{K}$. The problem is how to prove that we may assume $\mathbf{Q}(x$, $z) \subset \mathscr{K}$. In fact since $z$ is algebraic over $\mathscr{K}, \mathscr{O}_{P}(\mathcal{K}(z)) / m_{P}(\mathcal{K}(z))$ is finite algebraic over $\mathscr{O}_{p}(\mathcal{K}) / m_{p}(\mathcal{K})$ by [Z], Ch. VI, $\S 6$, Corollary 2 and hence consists of classical numbers by the argument of Corollary (3.2). We replace $\mathscr{K}$ of Definition (2.4) by

$$
\tilde{\mathscr{K}}=\mathscr{K}\left(z, \mathfrak{O}_{p}\left(\mathscr{K}(z) / m_{p}(\mathscr{K}(z))\right),\right.
$$

where we identify $O_{p}(\mathscr{K}(z)) / m_{p}(\mathscr{K}(z))$ canonically with a subfield of $\mathbf{C}$. Then $\widetilde{\mathcal{K}}$ consists of generalized $\mathbf{Q}$-classical functions and is finitely generated over $\mathbf{Q}(x)$. The point $P$ is a $C_{\mathscr{K}}\left(=\mathscr{O}_{P}(\mathscr{K}(z)) / m_{P}(\mathscr{K}(z))\right.$-rational point of $(D, \mathscr{K})$ by the generalized Lemma (2.3).

Once these lemmas are generalized, other results follow by a similar method.

When we defined $\mathbf{Q}$-classical functions, we allowed all the algebraic groups. Instead of doing this, we may restrict ourselves to one of the following categories of algebraic groups:

(1) the linear algebraic groups;

(2) the commutative linear algebraic groups;

(3) the commutative algebraic groups;

(4) the abelian varieties.

The corresponding fields of constants are denoted by $\mathbf{Q}^{\mathrm{li}}, \mathbf{Q}^{\mathrm{coli}}, \mathbf{Q}^{\mathrm{co}}$ and $\mathbf{Q}^{\mathrm{av}}$. These are subfields of $\mathbf{Q}^{\mathrm{cl}}$. The first three fields $\mathbf{Q}^{\mathrm{li}}, \mathbf{Q}^{\text {coli }}$ and $\mathbf{Q}^{\text {co }}$ are algebraically closed by the proof of Theorem (3.1) since in these cases the additive group $\mathbf{G}_{a \mathbf{Q}}$ is contained in the categories.

The last remark is that we can start from any subfield $F$ of $\mathbf{C}$ and define $F^{\text {cl }}$, $F^{\mathrm{li}}, F^{\mathrm{coli}}, F^{\mathrm{co}}$ and $F^{\mathrm{av}}$.

The following problem seems interesting in view of the solution of the algebraic equation by Theta constants (cf. [U1]) and Problem (6.12).

Problem (7.1). Is $\mathbf{Q}^{\text {av }}$ or more generally $F^{\text {av }}$ algebraically closed? 


\section{REFERENCES}

[B] Borel, A., Linear algebraic groups, Benjamin, New York, 1969.

[D] Dieudonné, J., Calcul infinitesimal, Collection Méthodes, Hermann, Paris, 1968.

[G] Gauss, C. F., Disquisitiones generales circa seriem infinitam $1+\frac{\alpha \beta}{1 \cdot \gamma} x+$ $\frac{\alpha(\alpha+1) \beta(\beta+1)}{1.2 \cdot \gamma(\gamma+1)} x x+\frac{\alpha(\alpha+1)(\alpha+2) \beta(\beta+1)(\beta+2)}{1.2 \cdot 3 \cdot \gamma(\gamma+1)(\gamma+2)} x^{3}+$ etc., pars prior, Gesamelte Werke Bd. III, 125-163, Göttingen, 1863-1933.

[H] Hartshorne, R., Algebraic geometry, Graduate. Texts. in Math., 52, SpringerVerlag, Berlin-Heidelberg-New York, 1977.

[K] Kolchin, E., Differential algebra and the algebraic groups, Academic Press, New York and London, 1973.

[L] Liouville, J., Sur la classification des transcendentes et sur l'impossibilité d'exprimer les racines de certaines équations en fonction finie explicite des coefficient, Journal de Math., 2 (1837), 56-104; 3 (1838), 523-546; 4 (1839), 423-456.

[M1] Mumford, D., Tata Lectures on Theta I, Progress in Math. Vol. 28, Birkhäuser, Boston-Basel-Stuttgart, 1983.

[M2] - The red book of varieties and schemes, Lec. Notes. in Math., Vol. 1358, Springer-Verlag, Berlin-Heidelberg-New York, 1988.

[P] Painlevé, P., Leçons de Stockholm, Oeuvres, Edition du C. N. R. S., Paris, 1972.

[R1] Rosenlicht, M., Some basic theorems on algebraic groups, Amer. J Math., 78 (1956), 401-443.

[R2] - Liouville's theorem on functions with elementary integrals, Pacific J. Math., 24 (1968), 153-161.

[S] Shafarevich, I. R., Basic algebraic geometry, Grundl. Math. Wiss., Bd. 213, Springer-Verlag, Berlin-Heidelberg-New York, 1974.

[U1] Umemura, H., Resolution of algebraic equations by theta constants, in D. Mumford: Tata Lectures on Theta II, Progress in Math. Vol. 43, Birkhäuser, Boston-Basel-Stuttgart, 1985.

[U2] - On the irreducibility of the first differential equation of Painlevé, Algebraic geometry and Commutative algebra in Honor of Masayoshi NAGATA, Kinokuniya 1987, Tokyo, 101-119.

[U3] - Second proof of the irreducibility of the first differential equation of Painlevé, Nagoya Math. J., 117 (1990), 125-171.

[U4] Birational automorphism groups and differential equations, Nagoya Math. J., 119 (1990), 1-80.

[W] Weyl, H., Die Idee der Riemannschen Fläche, Teubner, 1913.

[WW] Whittacker, E. T. and Watson, G. N., A course of modern analysis, Cambridge University Press, 1902.

[Z] Zariski, O. and Samuel, P., Commutative algebra, Van Nostrand, Princeton, 1985.

\section{Appendix}

This part is added to help the reader who is not familiar with algebraic geometry. Our aim here is not review rigorous definitions but to explain the termi- 
nology of algebraic geometry in a down-to-earth language.

(A1) We begin with conventions in algebraic geometry. Let $X$ be an algebraic variety defined over a field $k$. (If one wants to be rigorous, one should say that $X$ is a $k$-scheme of finite type ...etc.) We denote the structure sheaf of $X$ by $\mathscr{O}_{X}$ or simply by $\mathscr{O}$. So for a point $x \in X$ the fibre $\mathscr{O}_{x}$ is a local ring of the rational functions on $X$ which are regular at the point $x$ of $X$ (cf. [S], Chap. $V$ ). We denote by $m_{X}$ the maximal ideal of the local ring $\mathscr{O}_{x}$ so that $m_{x}=\left\{f \in \mathscr{O}_{x} \mid\right.$ the rational function $f$, which is regular in a neighbourhood of $x$, vanishes at $x\}$. We set $k(x)=$ $\mathscr{O}_{x} / m_{x}$ so that $k(x)$ is an over-field of $k$ and we call $k(x)$ the residue field of $x$ (cf. [H], Chap. II, Exercise 2.7).

(A2) Keeping the notation of (A1), we denote the $k$-vector space of the sections of $\mathscr{O}$ on $U$ by $\Gamma(U, \mathscr{O})$ or by $H^{0}(U, \mathscr{O})$ for an open set $U$ of $X$ so that $\Gamma(U, \mathscr{O})=H^{0}(U, \mathscr{O})$ is the ring of the rational functions of $X$ which are regular on $U$. Let $f: X \rightarrow Y$ be a morphism of algebraic varieties over $k$. So $f$ induces a $k$-linear map $\Gamma\left(Y, \mathscr{O}_{Y}\right) \rightarrow \Gamma\left(X, \mathscr{O}_{Y}\right)$ which we denote by $f^{*}$.

(A3) Let $X$ be as above. For a field extension $K / k$, the fibre product $X$ $\times_{\text {Spec } k} \operatorname{Spec} K$ will be denoted by $X \otimes_{k} K$ or by $X_{K}$ (cf. [S], Chap. $V$, $\S 4$, where a fibre product is simply called a product). So there is a canonical morphism $X_{K} \rightarrow$ $X$. If $X$ is an affine variety so that $X$ is a closed subset of the affine space $\mathbf{A}_{k}^{n}$ defined by a set of polynomials $f_{i}\left(x_{1}, x_{2}, \ldots, x_{n}\right) \in k\left[x_{1}, x_{2}, \ldots, x_{n}\right], 1 \leq i \leq m$. Since $k \subset K, f_{i}\left(x_{1}, x_{2}, \ldots, x_{n}\right) \in K\left[x_{1}, x_{2}, \ldots, x_{n}\right]$ and hence we can regard that $X \subset \mathbf{A}_{K}^{n}$ which is intuitively nothing but $X_{K}$. This alternative looks easy to understand but unfortunately is not convenient if we want to treat the varieties systematically. For this reason we have to define $X_{K}$ as $X \times_{\operatorname{Spec~} k} \operatorname{Spec} K$.

(A4) Let $X$ and $K / k$ be as above. A $K$-valued point of $X$ is a $k$-morphism Spec $K \rightarrow X$ of schemes.

(A4.1) We have a $1: 1$-correspondence between the elements of the following sets:

(i) The set of the $K$-valued points of $X$;

(ii) The set of the pairs $(x, \varphi)$, where $x$ is a point of $X$ and $\varphi$ is a $k$-morphism $\mathscr{O}_{x} / m_{x}=k(x) \rightarrow K$ of fields (cf. [H], Chap. II, Exercise 2.7).

Let $v: \operatorname{Spec} K \rightarrow X$ be a $K$-valued point so that a pair of a point $x \in X$ and a $k$-morphism $\varphi: \mathscr{O}_{x} / m_{x} \rightarrow K$ corresponds. Let $f$ be a rational function regular at $x$, i.e. $f \in \mathscr{O}_{x}$. Then we denote $\varphi(\bar{f})$ by $f(v)$ and call it the value of the function $f$ at the $K$-valued point $v$, where $\bar{f}$ is the class of $f$ in $\mathscr{O}_{x} / m_{x}$.

If these two equivalent conditions are difficult to understand, we can rephrase the definition as follows. Since the question is local, we may assume that $X$ is an affine variety so that $X$ is a closed subset for the Zariski topology of the affine 
space $\mathbf{A}_{k}^{n}$. Then giving a $K$-valued point is equivalent to give a point $x=\left(x_{1}, x_{2}\right.$, $\left.\ldots, x_{n}\right) \in X \subset \mathbf{A}_{k}^{n}$ whose coordinates $x_{i}(1 \leq i \leq n)$ are in $K$ since $k(x)=k\left(x_{1}\right.$, $\left.x_{2}, \ldots, x_{n}\right)$. We prefer however the previous definition because it is more convenient.

(A4.2) It follows from the definition of the fibre product $X_{K}$ that we have a canonical bijection between the elements of the following sets:

(i) The set of the $K$-valued points of $X$;

(ii) The set of the $K$-rational points of $X_{K}$. (cf. [S], Chap. V, §4 and [M2], Chap. II, §6).

(A5) There are a certain fixed notation for algebraic groups such as the general linear group is denoted by $\mathbf{G L}_{n k}$ when it is considered as an algebraic group defined over a field $k$. The affine line $\mathbf{A}_{k}^{1}$ with group law of adding the coordinates is called the additive group and denoted by $\mathbf{G}_{a k}$. The affine line $\mathbf{A}_{k}^{1}$ minus the origin with group law of multiplying the coordinates is called the multiplicative group and denoted by $\mathbf{G}_{m k}$ so that $\mathbf{G L}_{1 k}=\mathbf{G}_{m k}$. When there is no danger of confusing the reference field $k, \mathbf{G L}_{n k}, \mathbf{G}_{a k}, \mathbf{G}_{m k}$ are simply denoted by $\mathbf{G L}_{n}, \mathbf{G}_{a}$, $\mathbf{G}_{m}$. We refer the reader to Chap. I. of $[B]$.

(A6) A rational map or a meromorphic map is denoted by a dotted arrow.

(A7) For an algebraic variety $X$ defined over $\mathbf{C}$, the associated analytic space is denoted by $X^{\text {an }}$ (cf. [S], Chap. VIII).

Department of Mathematics

Nagoya University

Chikusa-ku, Nagoya 464-01

Japan 